\title{
THEORETICAL ASPECTS AND NUMERICAL COMPUTATION OF THE TIME-HARMONIC GREEN'S FUNCTION FOR AN ISOTROPIC ELASTIC HALF-PLANE WITH AN IMPEDANCE BOUNDARY CONDITION
}

\author{
Mario Durán ${ }^{1}$, Eduardo Godoy ${ }^{1}$ And Jean-Claude NÉdÉleC ${ }^{2}$
}

\begin{abstract}
This work presents an effective and accurate method for determining, from a theoretical and computational point of view, the time-harmonic Green's function of an isotropic elastic half-plane where an impedance boundary condition is considered. This method, based on the previous work done by Durán et al. (cf. [Numer. Math. 107 (2007) 295-314; IMA J. Appl. Math. 71 (2006) 853-876]) for the Helmholtz equation in a half-plane, combines appropriately analytical and numerical techniques, which has an important advantage because the obtention of explicit expressions for the surface waves. We show, in addition to the usual Rayleigh wave, another surface wave appearing in some special cases. Numerical results are given to illustrate that. This is an extended and detailed version of the previous article by Durán et al. [C. R. Acad. Sci. Paris, Ser. IIB 334 (2006) 725-731].
\end{abstract}

Mathematics Subject Classification. 31A10, 35E05, 65T50, 74B05, 74J15.

Received June 10, 2008. Revised June 18, 2009.

Published online February 23, 2010.

\section{INTRODUCTION}

The Green's functions for elastic half-spaces have been studied by many authors, because of their applicability in important areas of science and engineering such as seismology, geophysics, and structural engineering. In particular, the main motivation of the present study comes from a difficulty that arises in mining engineering, during the rock blasting in an underground mine. When the blasting devices are set out, the engineers attempt to take full advantage of the energy irradiated, but at the same time they desire to prevent the expansive wave from concentrating an important amount of energy at the exploitation areas of the mine, as serious damage could be caused. As a preliminary approach to this problem, we consider a linear isotropic elastic model, where the ground is represented as a half-plane and the underground mine can be described as a local perturbation of the boundary. Additionally, an impedance boundary condition, also named in related literature non-absorbing or passive boundary condition, is imposed in order to have a more general description of the surface dynamics. We propose that the computation of resonant states associated with this domain can provide a basic knowledge about the most probable directions that waves could take to radiate the energy generated.

\footnotetext{
Keywords and phrases. Green's function, half-plane, time-harmonic elasticity, impedance boundary condition, surface waves.

1 Facultad de Ingeniería, Pontificia Universidad Católica de Chile, Av. Vicuña Mackenna 4860, Macul, Santiago, Chile. mduran@ing.puc.cl; egodoy@ing.puc.cl

2 CMAP, École polytechnique, 91128 Palaiseau, France. nedelec@cmap.polytechnique.fr
} 
To compute numerically these resonant states, a boundary integral equations method is proposed. However, such methods require evaluation of boundary integrals containing the associated Green's function, hence the need of an efficient procedure for computing the Green's function, capable of yielding accurate enough expressions.

The standard method for obtaining half-space Green's functions employs application of Fourier or Laplace transforms in space to the partial differential equation of motion, as well as in time when dealing with transient problems. Systems of ordinary differential equations are achieved, which can be analytically solved, leading to the Green's function transferred to the Fourier or Laplace domain. This procedure has been widely used by many authors, nevertheless, the calculation of the inverse transforms in a numerically efficient way is not a simple matter, and there exist different approaches to this problem in related works. In 1974, Johnson [13] considered time-domain isotropic elasticity in a half-space and employed Cagniard-de Hoop inversion. The same method was applied in 1999 by Richter and Schmid [17] for a half-plane, including application to BEM. The Cagniard-de Hoop inversion has been proved to be a useful method for the transient case, but there is not direct application to time-harmonic problems. The case of layered half-spaces has been widely studied in seismology, and there are inversion methods specially adapted for this kind of solids. In 1983, Franssens [11] used a modified propagator matrix method for calculating the associated 2D Green's function. Another references about this kind of methods can be found in Chapter 4 of the book by Jensen et al. [12]. The anisotropic case has received more attention in recent years, in particular, general anisotropic half-spaces has been studied in 1996 by Wang and Achenbach [20] and in 1997 by Spies [18]. Wang and Achenbach did not deal with any Fourier or Laplace transforms but they constructed directly the solution by superposition of transient plane waves. The solution was expressed as integrals on finite domains, however, this method was limited to source points placed on the surface. Spies applied Fourier transforms in space and time, but no methods to calculate the inverse transforms were proposed. More recently, in 2007, Chen and Dravinski [4,5] considered time-harmonic Green's functions for a triclinic half-space, using a double Fourier transform. The inversion was made by employing contour integration and Gauss-Legendre quadrature.

In this paper we deal with an isotropic elastic half-plane and embedded point sources. Although the anisotropic case could be considered as more attractive due to its generality, the obtention of explicit expressions that characterize the surface waves becomes more difficult. As we will see, the impedance boundary condition has a direct influence on the surface waves that appear, and none of the works mentioned above deal with this kind of boundary condition. A slightly similar condition can be found in the paper by Linkov [15], where a model of the rupture process associated with a propagating crack was proposed. The Appendix of this work presents quite general interface conditions for two bonded elastic half-planes, including elastic, softening and viscous deformation. By neglecting one of the two half-planes, it is possible to approach our problem from another point of view.

The method used in the present work to compute the associated Green's function is based on recent works by Durán et al. [9], and Durán et al. [10] for the acoustic case with impedance boundary conditions. The half-plane Green's function of the Helmholtz's equation was theoretically determined in [9] and numerically implemented in [10]. The Fourier transform is applied only in the horizontal sense, and the spectral Green's function (that is, transferred to the Fourier domain) is expressed as a sum of two terms. The first one can be analytically inverted and it corresponds to the full-plane Green's function. The second term takes into account the particular effect of the half-plane and, in order to make an accurate computation of its inverse Fourier transform, it is decomposed into a sum of three new terms, where two of them contain singularities in the spectral variable (pseudo-poles and poles) and they can be analytically inverted, whereas the remaining term is regular, decreasing at infinity, and its inverse transform is numerically approximated via a backward FFT algorithm. In particular, the poles of the spectral Green's function have special significance, because each pair of poles is associated with the existence of a surface wave. These poles are determined from the roots of an algebraic equation, which in general has to be numerically solved. The well-known Rayleigh wave is obtained as a function of the impedance. Moreover, we have found that if the impedance takes a certain value, which only depends on the physical parameters of the elastic medium, a new surface wave appears. 


\section{BASIC MATHEMATICAL MODEL}

Let us consider a locally perturbed half-plane $\Omega_{e}$ representing the ground. Its boundary is denoted by $\Gamma$. We assume this domain to be constituted by an elastic, homogeneous, and isotropic medium. Let $\boldsymbol{u}: \Omega_{e} \longrightarrow \mathbb{C}^{2}$ be the displacement field of the perturbed half-plane, satisfying the time-harmonic elasticity equation:

$$
\operatorname{div} \sigma(\boldsymbol{u}(\boldsymbol{x}))+\rho \omega^{2} \boldsymbol{u}(\boldsymbol{x})=\mathbf{0} \quad \boldsymbol{x} \in \Omega_{e},
$$

where $\rho$ is the solid density and $\omega$ is a given pulsation or angular frequency. The stress tensor $\sigma$ is described by the isotropic Hooke's law:

$$
\sigma(\boldsymbol{u}(\boldsymbol{x}))=\lambda \operatorname{div} \boldsymbol{u}(\boldsymbol{x}) I+\mu\left(\nabla \boldsymbol{u}(\boldsymbol{x})+\nabla \boldsymbol{u}(\boldsymbol{x})^{T}\right),
$$

where $\lambda, \mu>0$ are the Lamé's constants, and $I$ is the $2 \times 2$ identity matrix. In a geophysical framework, the normal stresses on the ground surface are made equal to the atmospheric pressure exerted by the air, which can be neglected in practice, since it has no influence on the elastic waves that occur in the ground. Therefore, we assume null normal stresses on $\Gamma$, that is,

$$
\sigma(\boldsymbol{u}(\boldsymbol{x})) \boldsymbol{n} \cdot \boldsymbol{n}=0, \quad \boldsymbol{x} \in \Gamma
$$

where $\boldsymbol{n}$ denotes the unit outward normal vector to $\Gamma$. In addition, we could also suppose shear stresses to be null, obtaining a Neumann boundary condition (free-traction) on $\Gamma$. Alternatively, shear displacements could be set to zero, leading to a mixed boundary condition on $\Gamma$, which corresponds to a Neumann one in the normal sense and a Dirichlet one in the tangential sense. In the present work, we consider a Robin-type boundary condition in the tangential sense, which assumes that shear stresses are proportional to shear displacements on $\Gamma$. This assumption is mathematically expressed as

$$
\sigma(\boldsymbol{u}(\boldsymbol{x})) \boldsymbol{n} \cdot \boldsymbol{\tau}=\omega Z \boldsymbol{u}(\boldsymbol{x}) \cdot \boldsymbol{\tau} \quad \boldsymbol{x} \in \Gamma
$$

where $\boldsymbol{\tau}$ denotes the unit tangent vector to $\Gamma$ and $Z$ is the surface elastic impedance, which could be complex in general, but in the present work we treat only the case where $Z$ is real. From a physical point of view, as (1.4) establishes a linear relation between stresses and displacements, the impedance $Z$ can be regarded as a shear stiffness modulus associated with the surface. If $Z$ is negative, the surface behaves in a way similar to that of a spring-mass system, where forces are opposite to displacements, that is, a hardening phenomenon. On the contrary, if $Z$ is positive, we obtain that both quantities have the same sign, which can be assimilated to a surface softening phenomenon in the linear elastic range $(c f .[15])$. Furthermore, it is worth to remark that if $Z=0$, we retrieve the free-traction boundary condition, and if $|Z| \longrightarrow+\infty$, we approximate the mixed boundary condition mentioned above. Hence, our impedance boundary condition can be regarded as an intermediate case between these two. Both relations (1.3) and (1.4) can be written in vector form as

$$
-\sigma(\boldsymbol{u}(\boldsymbol{x})) \boldsymbol{n}+\omega Z(\boldsymbol{u}(\boldsymbol{x}) \cdot \boldsymbol{\tau}) \boldsymbol{\tau}=\mathbf{0} \quad \boldsymbol{x} \in \Gamma .
$$

Therefore, the time-harmonic elasticity system with an impedance boundary condition in a perturbed half-plane is expressed as follows:

$$
\begin{aligned}
\operatorname{div} \sigma(\boldsymbol{u}(\boldsymbol{x}))+\rho \omega^{2} \boldsymbol{u}(\boldsymbol{x}) & =\mathbf{0} & & \text { in } \Omega_{e}, \\
-\sigma(\boldsymbol{u}(\boldsymbol{x})) \boldsymbol{n}+\omega Z(\boldsymbol{u}(\boldsymbol{x}) \cdot \boldsymbol{\tau}) \boldsymbol{\tau} & =\boldsymbol{f}(\boldsymbol{x}) & & \text { on } \Gamma,
\end{aligned}
$$

+ Outgoing radiation conditions when $|\boldsymbol{x}| \longrightarrow+\infty$,

where a non-homogeneous data $f$ is added to the right-hand side of (1.6b). For the sake of simplicity, the function $\boldsymbol{f}$ is assumed to have compact support. When determining resonant states, $\boldsymbol{f}$ is set to zero and we look for complex numbers $\omega$ (the resonances) and non-zero displacement fields $\boldsymbol{u}$ (the resonant states) satisfying (1.6). Additionally, radiation conditions at infinity have to be prescribed in order to ensure mathematical 
well-posedness of the model. From a physical point of view, these radiation conditions eliminate solutions that correspond to incoming waves, allowing only outgoing waves, which have the right physical sense. The obtention of an explicit form for the radiation conditions associated with (1.6) constitutes a complex matter, and it is beyond the scope of the present work.

To solve (1.6) by integral equation techniques, it is necessary to know the associated Green's function in the non-perturbed half-plane. By convention, we consider the upper half-plane $\mathbb{R}_{+}^{2}$, defined as

$$
\mathbb{R}_{+}^{2}=\left\{\boldsymbol{x}=\left(x_{1}, x_{2}\right) \in \mathbb{R}^{2} / x_{2}>0\right\} .
$$

Notice that in this case, vectors $\boldsymbol{n}$ and $\boldsymbol{\tau}$ become $(0,-1)$ and $(1,0)$, respectively. Let $\boldsymbol{x}$ and $\boldsymbol{y}$ be the receiver and source points, respectively, with $\boldsymbol{x}, \boldsymbol{y} \in \mathbb{R}_{+}^{2}$. The Green's function of (1.6), denoted by $G=G(\boldsymbol{x}, \boldsymbol{y})$, is a $2 \times 2$ matrix function with complex values. Its column vectors, denoted by $\boldsymbol{g}_{j}=\boldsymbol{g}_{j}(\boldsymbol{x}, \boldsymbol{y})(j=1,2)$, must satisfy

$$
\begin{array}{rlrl}
\operatorname{div}_{\boldsymbol{x}} \sigma_{\boldsymbol{x}}\left(\boldsymbol{g}_{j}(\boldsymbol{x}, \boldsymbol{y})\right)+\rho \omega^{2} \boldsymbol{g}_{j}(\boldsymbol{x}, \boldsymbol{y}) & =-\delta(\boldsymbol{x}-\boldsymbol{y}) \hat{\boldsymbol{e}}_{j} & & \text { in } \mathbb{R}_{+}^{2}, \\
\sigma_{\boldsymbol{x}}\left(\boldsymbol{g}_{j}(\boldsymbol{x}, \boldsymbol{y})\right) \hat{\boldsymbol{e}}_{2}+\omega Z\left(\boldsymbol{g}_{j}(\boldsymbol{x}, \boldsymbol{y}) \cdot \hat{\boldsymbol{e}}_{1}\right) \hat{\boldsymbol{e}}_{1}=\mathbf{0} & & \text { on }\left\{x_{2}=0\right\},
\end{array}
$$

+ Outgoing radiation conditions when $|x| \longrightarrow+\infty$,

where $\delta(\boldsymbol{x}-\boldsymbol{y})$ is the Dirac mass evaluated at $\boldsymbol{x}$ and centered at $\boldsymbol{y}$, and $\hat{\boldsymbol{e}}_{j}$ is the $j$ th canonic vector of $\mathbb{R}^{2}$. The variable $\boldsymbol{x}$ is added as a subindex in order to emphasize that all the involved derivatives are with respect to $\boldsymbol{x}$.

For a broader framework about Green's functions and their use in integral equations for time-harmonic problems, see Bonnet [3], Colton and Kress [6], Linkov [14], and Nédélec [16].

\section{Spectral Green's Function}

To solve system (1.8), a partial Fourier transform in the horizontal variable $x_{1}$ is applied, which uses a special Fourier variable $s$ normalized by $\omega$. Let us denote this transform by $\mathcal{F}_{\omega}$, defined as

$$
\begin{aligned}
& \Phi\left(s, x_{2}\right)=\left[\mathcal{F}_{\omega} \phi\right]\left(s, x_{2}\right)=\int_{-\infty}^{+\infty} \phi\left(x_{1}, x_{2}\right) \mathrm{e}^{-\mathrm{i} \omega s\left(x_{1}-y_{1}\right)} \mathrm{d} x_{1}, \\
& \phi\left(x_{1}, x_{2}\right)=\left[\mathcal{F}_{\omega}^{-1} \Phi\right]\left(x_{1}, x_{2}\right)=\frac{\omega}{2 \pi} \int_{-\infty}^{+\infty} \Phi\left(s, x_{2}\right) \mathrm{e}^{\mathrm{i} \omega s\left(x_{1}-y_{1}\right)} \mathrm{d} s
\end{aligned}
$$

where for the sake of simplicity, the dependence on the source point $\boldsymbol{y}$ is not explicitly written for the time being. Applying $\mathcal{F}_{\omega}$ to system (1.8) both for $j=1$ and $j=2$ yields

$$
\begin{gathered}
C_{22} \frac{\partial^{2} \widehat{G}}{\partial x_{2}^{2}}\left(s, x_{2}\right)+\mathrm{i} \omega s\left(C_{12}+C_{21}\right) \frac{\partial \widehat{G}}{\partial x_{2}}\left(s, x_{2}\right)-\omega^{2}\left(s^{2} C_{11}-\rho I\right) \widehat{G}\left(s, x_{2}\right)=-\delta\left(x_{2}-y_{2}\right) I, \\
C_{22} \frac{\partial \widehat{G}}{\partial x_{2}}(s, 0)+\omega\left(\text { is } C_{21}+Z I_{1}\right) \widehat{G}(s, 0)=0,
\end{gathered}
$$

which corresponds to a matrix ODE's system for $\widehat{G}=\mathcal{F}_{\omega} G$. The matrices $C_{j l}$ are defined by

$$
C_{j l}=\left[\begin{array}{ll}
c_{1 j 1 l} & c_{1 j 2 l} \\
c_{2 j 1 l} & c_{2 j 2 l}
\end{array}\right], \quad 1 \leq j, l \leq 2
$$


where the coefficients $c_{i j k l}$ are given by

$$
c_{i j k l}=\left\{\begin{array}{cl}
\lambda+2 \mu & \text { if } i=j=k=l \\
\lambda & \text { if } i=j \text { and } k=l \neq i, \\
\mu & \text { if } i=k \text { and } j=l \neq i, \\
\mu & \text { if } i=l \text { and } j=k \neq i, \\
0 & \text { in any other case }
\end{array}\right.
$$

and $I_{1}=\hat{\boldsymbol{e}}_{1} \hat{\boldsymbol{e}}_{1}^{T}$. The matrix function $\widehat{G}$ denotes the spectral Green's function. As it was done in [9] for the Helmholtz equation, we attempt to express $\widehat{G}$ as a sum of two terms:

$$
\widehat{G}\left(s, x_{2}\right)=\widehat{G}^{P}\left(s, x_{2}\right)+\widehat{G}^{B}\left(s, x_{2}\right),
$$

where $\widehat{G}^{P}$ is a term associated with the full-plane and $\widehat{G}^{B}$ is a term that takes into account the half-plane's surface and the boundary condition considered. In order to calculate the solution to (2.2), we start by solving the homogeneous equation of (2.2a) on both sides $\left\{0 \leq x_{2} \leq y_{2}\right\}$ and $\left\{x_{2} \geq y_{2}\right\}$. After that, suitable transmission conditions are imposed at $x_{2}=y_{2}$. Notice that if the right-hand side of (2.2a) is set to zero, then the differential equation becomes the same for both columns of $\widehat{G}$, so it is possible to write the homogeneous equation in vectorial form:

$$
C_{22} \boldsymbol{r}^{\prime \prime}\left(x_{2}\right)+\mathrm{i} \omega s\left(C_{12}+C_{21}\right) \boldsymbol{r}^{\prime}\left(x_{2}\right)-\omega^{2}\left(s^{2} C_{11}-\rho I\right) \boldsymbol{r}\left(x_{2}\right)=\mathbf{0},
$$

where $s$ is assumed to be a parameter. Any solution of (2.6) can be expressed as a linear combination of terms of the form

$$
\boldsymbol{r}\left(x_{2}\right)=\boldsymbol{w} \mathrm{e}^{-\mathrm{i} \omega \nu x_{2}},
$$

where $\nu \in \mathbb{C}$ is a scalar and $\boldsymbol{w} \in \mathbb{C}^{2}$ is a vector, which are unknowns and not depending on $\boldsymbol{y}$. Substituting (2.7) in (2.6) and expanding, leads to a characteristic equation:

$$
A(s, \nu) \boldsymbol{w}=\mathbf{0},
$$

where

$$
A(s, \nu)=\nu^{2} C_{22}-\nu s\left(C_{21}+C_{12}\right)+s^{2} C_{11}-\rho I .
$$

Thus, the pairs $(\nu, \boldsymbol{w})$ in (2.7) are the non-trivial solutions of the characteristic equation (2.8). In particular, the scalars $\nu$ have to be such that $A$ is singular, that is,

$$
\operatorname{det} A(s, \nu)=0 .
$$

Replacing (2.9) in (2.10) and expanding, leads to a polynomial equation for $\nu$ :

$$
\left(\nu^{2}+\left(s^{2}-s_{L}^{2}\right)\right)\left(\nu^{2}+\left(s^{2}-s_{T}^{2}\right)\right)=0,
$$

where $s_{L}$ and $s_{T}$ are the longitudinal and transversal slownesses, respectively, defined by

$$
s_{L}=\sqrt{\frac{\rho}{\lambda+2 \mu}}, \quad s_{T}=\sqrt{\frac{\rho}{\mu}},
$$

which satisfy $s_{L}<s_{T}$. Equation (2.10) has four independent solutions. They are

$$
\nu_{L^{+}}=\mathrm{i} \theta_{L}(s), \quad \nu_{L^{-}}=-\mathrm{i} \theta_{L}(s), \quad \nu_{T^{+}}=\mathrm{i} \theta_{T}(s), \quad \nu_{T^{-}}=-\mathrm{i} \theta_{T}(s),
$$

where $\theta_{L}(\cdot)$ and $\theta_{T}(\cdot)$ are the following functions:

$$
\theta_{L}(s)=\sqrt{s^{2}-s_{L}^{2}}, \quad \theta_{T}(s)=\sqrt{s^{2}-s_{T}^{2}} .
$$




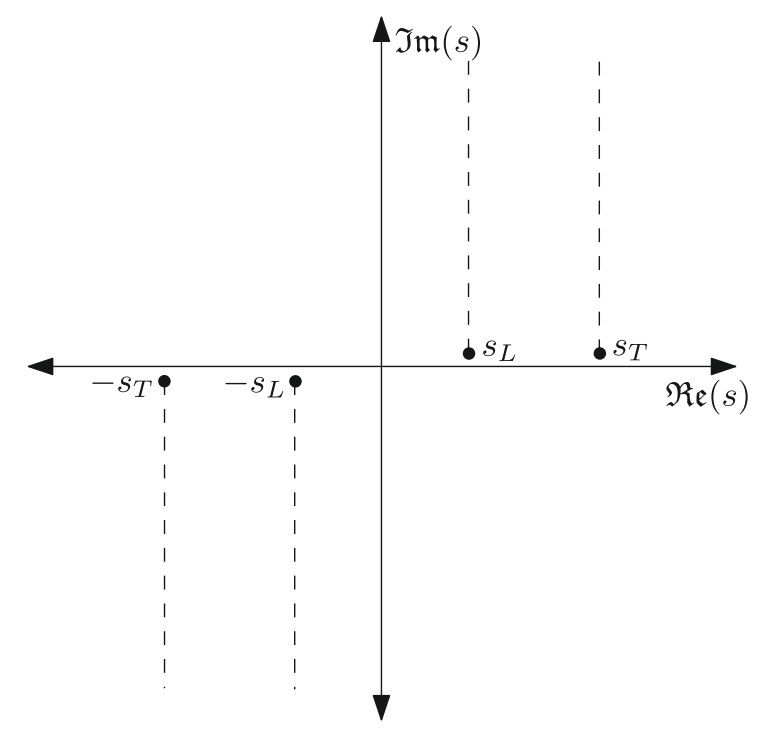

Figure 1. Domain of complex maps $\theta_{L}(\cdot)$ and $\theta_{T}(\cdot)$.

Even though $s \in \mathbb{R}$, these are complex maps, therefore, an exact meaning has to be given to the square roots. We put

$$
\theta_{L}(s)=\sqrt{s-s_{L}} \sqrt{s+s_{L}}, \quad \theta_{T}(s)=\sqrt{s-s_{T}} \sqrt{s+s_{T}}
$$

and we consider particular branches in the complex plane to define each root, as indicated in Figure 1 . The exact definitions are:

$$
\theta_{L}(s)=-\mathrm{i} s_{L} \exp \left(\int_{0}^{s} \frac{u}{u^{2}-s^{2}} \mathrm{~d} u\right), \quad \theta_{T}(s)=-\mathrm{i} s_{T} \exp \left(\int_{0}^{s} \frac{u}{u^{2}-s^{2}} \mathrm{~d} u\right) .
$$

When dealing with these definitions, $\theta_{L}(s)$ and $\theta_{T}(s)$ have always non negative real part for $s \in \mathbb{R}$ (for more details, see $[9,10])$. Then, the vectors $\boldsymbol{w}$ are computed by substituting the associated values $\nu$ in $(2.8)$ :

$$
\boldsymbol{w}_{L^{+}}=\left[\begin{array}{c}
\mathrm{i} s \\
\theta_{L}(s)
\end{array}\right], \quad \boldsymbol{w}_{L^{-}}=\left[\begin{array}{c}
-\mathrm{i} s \\
\theta_{L}(s)
\end{array}\right], \quad \boldsymbol{w}_{T^{+}}=\left[\begin{array}{c}
\theta_{T}(s) \\
-\mathrm{i} s
\end{array}\right], \quad \boldsymbol{w}_{T^{-}}=\left[\begin{array}{c}
\theta_{T}(s) \\
\mathrm{i} s
\end{array}\right] .
$$

The general solution of (2.6) can be expressed as a linear combination:

$$
\boldsymbol{r}\left(x_{2}\right)=\alpha_{L^{+}} \mathrm{e}^{-\mathrm{i} \omega \nu_{L^{+}} x_{2}} \boldsymbol{w}_{L^{+}}+\alpha_{L^{-}} \mathrm{e}^{-\mathrm{i} \omega \nu_{L^{-}} x_{2}} \boldsymbol{w}_{L^{-}}+\alpha_{T^{+}} \mathrm{e}^{-\mathrm{i} \omega \nu_{T^{+}} x_{2}} \boldsymbol{w}_{T^{+}}+\alpha_{T^{-}} \mathrm{e}^{-\mathrm{i} \omega \nu_{T^{-}} x_{2}} \boldsymbol{w}_{T^{-}},
$$

for general complex coefficients $\alpha_{L^{+}}, \alpha_{L^{-}}, \alpha_{T^{+}}$, and $\alpha_{T^{-}}$. Moreover, $r\left(x_{2}\right)$ has to verify a boundary condition at $x_{2}=0$, which can be easily obtained from $(2.2 \mathrm{~b})$ :

$$
C_{22} \boldsymbol{r}^{\prime}(0)+\omega\left(\text { is } C_{21}+Z I_{1}\right) \boldsymbol{r}(0)=\mathbf{0} .
$$

Replacing $\boldsymbol{r}\left(x_{2}\right)$ from (2.17) in (2.18) leads to the next identity:

$$
\alpha_{L^{+}} \boldsymbol{v}_{L^{+}}+\alpha_{L^{-}} \boldsymbol{v}_{L^{-}}+\alpha_{T^{+}} \boldsymbol{v}_{T^{+}}+\alpha_{T^{-}} \boldsymbol{v}_{T^{-}}=0
$$

where the vectors $\boldsymbol{v}_{j}$ are obtained from the vectors $\boldsymbol{w}_{j}\left(j=L^{+}, L^{-}, T^{+}, T^{-}\right)$by means of the relation

$$
\boldsymbol{v}_{j}=\left(\nu_{j} C_{22}-s C_{21}+\mathrm{i} Z I_{1}\right) \boldsymbol{w}_{j}
$$


Substituting (2.13) and (2.16) in (2.20) yields

$$
\begin{array}{rlrl}
\boldsymbol{v}_{L^{+}} & =\left[\begin{array}{c}
-s\left(2 \mu \theta_{L}(s)+Z\right) \\
\mathrm{i} \mu\left(s^{2}+\theta_{T}(s)^{2}\right)
\end{array}\right], & \boldsymbol{v}_{L^{-}} & =\left[\begin{array}{c}
-s\left(2 \mu \theta_{L}(s)-Z\right) \\
-\mathrm{i} \mu\left(s^{2}+\theta_{T}(s)^{2}\right)
\end{array}\right], \\
\boldsymbol{v}_{T^{+}}=\left[\begin{array}{c}
\mathrm{i} \mu\left(s^{2}+\theta_{T}(s)^{2}\right)+\mathrm{i} \theta_{T}(s) Z \\
2 \mu s \theta_{T}(s)
\end{array}\right], & \boldsymbol{v}_{T^{-}}=\left[\begin{array}{c}
-\mathrm{i} \mu\left(s^{2}+\theta_{T}(s)^{2}\right)+\mathrm{i} \theta_{T}(s) Z \\
2 \mu s \theta_{T}(s)
\end{array}\right] .
\end{array}
$$

As each column of $\widehat{G}$ is expressed in the form (2.17), we use a concise notation to write $\widehat{G}$ directly. For this, we define the matrices

which fulfill the properties

$$
W_{+}=\left[\boldsymbol{w}_{L^{+}} \mid \boldsymbol{w}_{T^{+}}\right], \quad W_{-}=\left[\boldsymbol{w}_{L^{-}} \mid \boldsymbol{w}_{T^{-}}\right]
$$

$$
W_{ \pm}^{-1}=-\frac{1}{\operatorname{det} W_{ \pm}} W_{ \pm}, \quad \operatorname{det} W_{+}=\operatorname{det} W_{-} .
$$

Additionally, we define the diagonal matrix

$$
D\left(x_{2}\right)=\operatorname{diag}\left[\mathrm{e}^{\omega \theta_{L}(s) x_{2}}, \mathrm{e}^{\omega \theta_{T}(s) x_{2}}\right],
$$

which satisfies

$$
D\left(x_{2}\right)=\operatorname{diag}\left[\mathrm{e}^{-\mathrm{i} \omega \nu_{L}+x_{2}}, \mathrm{e}^{-\mathrm{i} \omega \nu_{T^{+}} x_{2}}\right], \quad D\left(-x_{2}\right)=\operatorname{diag}\left[\mathrm{e}^{-\mathrm{i} \omega \nu_{L^{-}} x_{2}}, \mathrm{e}^{-\mathrm{i} \omega \nu_{T^{-}} x_{2}}\right] .
$$

The spectral Green's function $\widehat{G}$ is then expressed as follows:

$$
\widehat{G}\left(s, x_{2}\right)= \begin{cases}W_{+} D\left(x_{2}\right) A_{+}+W_{-} D\left(-x_{2}\right) A_{-} & \text {if } 0 \leq x_{2} \leq y_{2} \\ W_{+} D\left(x_{2}\right) B_{+}+W_{-} D\left(-x_{2}\right) B_{-} & \text {if } x_{2} \geq y_{2}\end{cases}
$$

where $A_{+}, A_{-}, B_{+}$, and $B_{-}$are unknowns matrices depending on $s$ and $y_{2}$. These matrices are determined by using the radiation conditions when $x_{2}$ tends to infinity, the transmissions conditions at $x_{2}=y_{2}$, and the boundary condition at $x_{2}=0$ given by (2.19). Let us begin by analyzing the case when $x_{2} \longrightarrow+\infty$. If $|s|>s_{L}$, then $\theta_{L}(s)$ is real, positive and the functions $\mathrm{e}^{\omega \theta_{L}(s) x_{2}}$ and $\mathrm{e}^{-\omega \theta_{L}(s) x_{2}}$ are exponentially increasing and decreasing in $x_{2}$, respectively. On the contrary, if $|s|<s_{L}$, then $\theta_{L}(s)$ is purely imaginary and both the above exponential functions behave oscillatorily in $x_{2}$. Indeed, it can be easily verified that in accordance with our definition of complex square roots, the imaginary part of $\theta_{L}(s)$ is negative. Consequently, $\mathrm{e}^{\omega \theta_{L}(s) x_{2}}$ contains waves that travel in the $-x_{2}$ sense, that is, incoming terms, whereas $\mathrm{e}^{-\omega \theta_{L}(s) x_{2}}$ contains waves that travel in the $+x_{2}$ sense, that is, outgoing terms. The analysis for the functions $\theta_{T}(s), \mathrm{e}^{\omega \theta_{T}(s) x_{2}}$ and $\mathrm{e}^{-\omega \theta_{T}(s) x_{2}}$ is completely analogous. We want to eliminate any exponentially increasing term in $x_{2}$, because it has no physical meaning, and all the incoming terms, in order to fulfill the radiation conditions at infinity. It is easy to observe that all these undesired behaviors occur in the matrix $D\left(x_{2}\right)$ (see (2.24) and (2.26)), therefore, it is natural to set

$$
B_{+}=0,
$$

and then we only keep the terms that behave as desired, which are contained within the matrix $D\left(-x_{2}\right)$. Let us study now the transmission conditions at $x_{2}=y_{2}$. We assume $\widehat{G}$ to be continuous at $x_{2}=y_{2}$, that is,

$$
W_{-} D\left(-y_{2}\right) B_{-}=W_{+} D\left(y_{2}\right) A_{+}+W_{-} D\left(-y_{2}\right) A_{-} .
$$

However, $\widehat{G}$ is not differentiable at this point. Then, its first derivative has a jump at $x_{2}=y_{2}$, given by

$$
\left[\frac{\partial \widehat{G}}{\partial x_{2}}\right]\left(s, y_{2}\right)=\lim _{x_{2} \rightarrow y_{2}^{+}} \frac{\partial \widehat{G}}{\partial x_{2}}\left(s, x_{2}\right)-\lim _{x_{2} \rightarrow y_{2}^{-}} \frac{\partial \widehat{G}}{\partial x_{2}}\left(s, x_{2}\right) .
$$


Computing the derivatives from (2.26), replacing (2.22) and combining with (2.28), (2.14) and (2.12) yields

$$
\left[\frac{\partial \widehat{G}}{\partial x_{2}}\right]\left(s, y_{2}\right)=-\frac{2 \rho \omega}{\operatorname{det} W_{+}} C_{22}^{-1} N W_{+} D\left(y_{2}\right) A_{+},
$$

where

$$
N=\operatorname{diag}\left[\theta_{L}(s), \theta_{T}(s)\right] .
$$

Consequently, the second derivative of $\widehat{G}$ in the sense of distributions corresponds to a Dirac mass centered at $y_{2}$ and multiplied by the jump of the derivative of $\widehat{G}$ at $x_{2}=y_{2}$. Substituting $\widehat{G}$ from (2.26) in (2.2a) and combining with (2.23), (2.28) and (2.30) gives $A_{+}$:

$$
A_{+}=-\frac{1}{2 \rho \omega} D\left(-y_{2}\right) W_{+} N^{-1} .
$$

Notice that at the moment, we have the solution to (2.2a) (taking into account the right-hand side). Finally, the boundary condition at $x_{2}=0$ given by (2.19) is imposed to each column of $\widehat{G}$, obtaining:

$$
V_{+} A_{+}+V_{-} A_{-}=0
$$

where the matrices $V_{+}$and $V_{-}$are defined from the vectors $\boldsymbol{v}_{L^{+}}, \boldsymbol{v}_{L^{-}}, \boldsymbol{v}_{T^{+}}$, and $\boldsymbol{v}_{T^{-}}$as follows:

$$
V_{+}=\left[\boldsymbol{v}_{L^{+}} \mid \boldsymbol{v}_{T^{+}}\right], \quad V_{-}=\left[\boldsymbol{v}_{L^{-}} \mid \boldsymbol{v}_{T^{-}}\right]
$$

Then, replacing (2.32) in (2.33), we determine $A_{-}$:

$$
A_{-}=\frac{1}{2 \rho \omega} V_{-}^{-1} V_{+} D\left(-y_{2}\right) W_{+} N^{-1}
$$

and substituting (2.32) and (2.35) in (2.28) gives $B_{-}$:

$$
B_{-}=-\frac{1}{2 \rho \omega}\left(D\left(y_{2}\right) W_{-}-V_{-}^{-1} V_{+} D\left(-y_{2}\right) W_{+}\right) N^{-1} .
$$

After that, replacing (2.27), (2.32), (2.35), and (2.36) in (2.26), the terms of the sum in (2.5) can be determined. The first term $\widehat{G}^{P}$ is given by

$$
\widehat{G}^{P}\left(s, x_{2}\right)= \begin{cases}-\frac{1}{2 \rho \omega} W_{+} D\left(x_{2}-y_{2}\right) W_{+} N^{-1} & \text { if } 0 \leq x_{2} \leq y_{2}, \\ -\frac{1}{2 \rho \omega} W_{-} D\left(y_{2}-x_{2}\right) W_{-} N^{-1} & \text { if } x_{2} \geq y_{2},\end{cases}
$$

and the second term $\widehat{G}^{B}$ is given by

$$
\widehat{G}^{B}\left(s, x_{2}\right)=\frac{1}{2 \rho \omega} W_{-} D\left(-x_{2}\right) V_{-}^{-1} V_{+} D\left(-y_{2}\right) W_{+} N^{-1} .
$$


Replacing $(2.22),(2.24)$, and (2.31) in (2.37), yields $\widehat{G}^{P}$, which is a symmetric matrix, whose components are

$$
\begin{aligned}
& \widehat{G}_{11}^{P}\left(s, x_{2}\right)=\frac{1}{2 \rho \omega}\left(s^{2} \theta_{L}(s)^{-1} \mathrm{e}^{-\omega \theta_{L}(s)\left|x_{2}-y_{2}\right|}-\theta_{T}(s) \mathrm{e}^{-\omega \theta_{T}(s)\left|x_{2}-y_{2}\right|}\right), \\
& \widehat{G}_{12}^{P}\left(s, x_{2}\right)=\frac{\mathrm{i} s}{2 \rho \omega} \operatorname{sign}\left(x_{2}-y_{2}\right)\left(\mathrm{e}^{-\omega \theta_{L}(s)\left|x_{2}-y_{2}\right|}-\mathrm{e}^{-\omega \theta_{T}(s)\left|x_{2}-y_{2}\right|}\right), \\
& \widehat{G}_{22}^{P}\left(s, x_{2}\right)=-\frac{1}{2 \rho \omega}\left(\theta_{L}(s) \mathrm{e}^{-\omega \theta_{L}(s)\left|x_{2}-y_{2}\right|}-s^{2} \theta_{T}(s)^{-1} \mathrm{e}^{-\omega \theta_{T}(s)\left|x_{2}-y_{2}\right|}\right) .
\end{aligned}
$$

From now on we call this term full-plane term. On the other hand, substituting (2.22), (2.34), (2.24), and (2.31) in (2.38), we obtain the components of $\widehat{G}^{B}$ :

$$
\begin{aligned}
& \widehat{G}_{11}^{B}\left(s, x_{2}\right)=-\frac{1}{2 \rho \omega}\left(\left(2 s^{2}-s_{T}^{2}\right)^{2}-4 s^{2} \theta_{L}(s) \theta_{T}(s)+\mu^{-1} s_{T}^{2} \theta_{T}(s) Z\right)^{-1} \\
& \times\left(s^{2} \theta_{L}(s)^{-1}\left(\left(2 s^{2}-s_{T}^{2}\right)^{2}+4 s^{2} \theta_{L}(s) \theta_{T}(s)+\mu^{-1} s_{T}^{2} \theta_{T}(s) Z\right) \mathrm{e}^{-\omega \theta_{L}(s)\left(x_{2}+y_{2}\right)}\right. \\
& +\theta_{T}(s)\left(\left(2 s^{2}-s_{T}^{2}\right)^{2}+4 s^{2} \theta_{L}(s) \theta_{T}(s)-\mu^{-1} s_{T}^{2} \theta_{T}(s) Z\right) \mathrm{e}^{-\omega \theta_{T}(s)\left(x_{2}+y_{2}\right)} \\
& \left.-4 s^{2}\left(2 s^{2}-s_{T}^{2}\right) \theta_{T}(s)\left(\mathrm{e}^{-\omega\left(\theta_{L}(s) x_{2}+\theta_{T}(s) y_{2}\right)}+\mathrm{e}^{-\omega\left(\theta_{T}(s) x_{2}+\theta_{L}(s) y_{2}\right)}\right)\right) \text {, } \\
& \widehat{G}_{21}^{B}\left(s, x_{2}\right)=-\frac{\mathrm{i} s}{2 \rho \omega}\left(\left(2 s^{2}-s_{T}^{2}\right)^{2}-4 s^{2} \theta_{L}(s) \theta_{T}(s)+\mu^{-1} s_{T}^{2} \theta_{T}(s) Z\right)^{-1} \\
& \times\left(\left(\left(2 s^{2}-s_{T}^{2}\right)^{2}+4 s^{2} \theta_{L}(s) \theta_{T}(s)+\mu^{-1} s_{T}^{2} \theta_{T}(s) Z\right) \mathrm{e}^{-\omega \theta_{L}(s)\left(x_{2}+y_{2}\right)}\right. \\
& +\left(\left(2 s^{2}-s_{T}^{2}\right)^{2}+4 s^{2} \theta_{L}(s) \theta_{T}(s)-\mu^{-1} s_{T}^{2} \theta_{T}(s) Z\right) \mathrm{e}^{-\omega \theta_{T}(s)\left(x_{2}+y_{2}\right)} \\
& \left.-4\left(2 s^{2}-s_{T}^{2}\right)\left(\theta_{L}(s) \theta_{T}(s) \mathrm{e}^{-\omega\left(\theta_{L}(s) x_{2}+\theta_{T}(s) y_{2}\right)}+s^{2} \mathrm{e}^{-\omega\left(\theta_{T}(s) x_{2}+\theta_{L}(s) y_{2}\right)}\right)\right) \text {, } \\
& \widehat{G}_{12}^{B}\left(s, x_{2}\right)=\frac{\mathrm{i} s}{2 \rho \omega}\left(\left(2 s^{2}-s_{T}^{2}\right)^{2}-4 s^{2} \theta_{L}(s) \theta_{T}(s)+\mu^{-1} s_{T}^{2} \theta_{T}(s) Z\right)^{-1} \\
& \times\left(\left(\left(2 s^{2}-s_{T}^{2}\right)^{2}+4 s^{2} \theta_{L}(s) \theta_{T}(s)+\mu^{-1} s_{T}^{2} \theta_{T}(s) Z\right) \mathrm{e}^{-\omega \theta_{L}(s)\left(x_{2}+y_{2}\right)}\right. \\
& +\left(\left(2 s^{2}-s_{T}^{2}\right)^{2}+4 s^{2} \theta_{L}(s) \theta_{T}(s)-\mu^{-1} s_{T}^{2} \theta_{T}(s) Z\right) \mathrm{e}^{-\omega \theta_{T}(s)\left(x_{2}+y_{2}\right)} \\
& \left.-4\left(2 s^{2}-s_{T}^{2}\right)\left(s^{2} \mathrm{e}^{-\omega\left(\theta_{L}(s) x_{2}+\theta_{T}(s) y_{2}\right)}+\theta_{L}(s) \theta_{T}(s) \mathrm{e}^{-\omega\left(\theta_{T}(s) x_{2}+\theta_{L}(s) y_{2}\right)}\right)\right) \text {, } \\
& \widehat{G}_{22}^{B}\left(s, x_{2}\right)=-\frac{1}{2 \rho \omega}\left(\left(2 s^{2}-s_{T}^{2}\right)^{2}-4 s^{2} \theta_{L}(s) \theta_{T}(s)+\mu^{-1} s_{T}^{2} \theta_{T}(s) Z\right)^{-1} \\
& \times\left(\theta_{L}(s)\left(\left(2 s^{2}-s_{T}^{2}\right)^{2}+4 s^{2} \theta_{L}(s) \theta_{T}(s)+\mu^{-1} s_{T}^{2} \theta_{T}(s) Z\right) \mathrm{e}^{-\omega \theta_{L}(s)\left(x_{2}+y_{2}\right)}\right. \\
& +s^{2} \theta_{T}(s)^{-1}\left(\left(2 s^{2}-s_{T}^{2}\right)^{2}+4 s^{2} \theta_{L}(s) \theta_{T}(s)-\mu^{-1} s_{T}^{2} \theta_{T}(s) Z\right) \mathrm{e}^{-\omega \theta_{T}(s)\left(x_{2}+y_{2}\right)} \\
& \left.-4 s^{2}\left(2 s^{2}-s_{T}^{2}\right) \theta_{L}(s)\left(\mathrm{e}^{-\omega\left(\theta_{L}(s) x_{2}+\theta_{T}(s) y_{2}\right)}+\mathrm{e}^{-\omega\left(\theta_{T}(s) x_{2}+\theta_{L}(s) y_{2}\right)}\right)\right) .
\end{aligned}
$$

Henceforth we call this term boundary term. 


\section{Effective calculation of Green's function}

In order to obtain an effective expression for the desired Green's function $G(\boldsymbol{x}, \boldsymbol{y})$, it is necessary to calculate accurately the inverse Fourier transform of the spectral Green's function $\widehat{G}\left(s, x_{2}\right)$, given by

$$
G(\boldsymbol{x}, \boldsymbol{y})=\frac{\omega}{2 \pi} \int_{-\infty}^{+\infty} \widehat{G}\left(s, x_{2}\right) \mathrm{e}^{\mathrm{i} \omega s\left(x_{1}-y_{1}\right)} \mathrm{d} s .
$$

This computation is made separately for the terms $\widehat{G}^{P}\left(s, x_{2}\right)$ and $\widehat{G}^{B}\left(s, x_{2}\right)$ obtained in the previous section. Furthermore, substituting (2.39) and (2.40) in (3.1), it is possible to verify a priori that the components of the Green's function fulfill the symmetries

$$
\begin{array}{ll}
G_{11}(\boldsymbol{x}, \boldsymbol{y})=G_{11}(\boldsymbol{y}, \boldsymbol{x}), & G_{12}(\boldsymbol{x}, \boldsymbol{y})=G_{21}(\boldsymbol{y}, \boldsymbol{x}), \\
G_{21}(\boldsymbol{x}, \boldsymbol{y})=G_{12}(\boldsymbol{y}, \boldsymbol{x}), & G_{22}(\boldsymbol{x}, \boldsymbol{y})=G_{22}(\boldsymbol{y}, \boldsymbol{x}),
\end{array}
$$

for all $\boldsymbol{x}, \boldsymbol{y} \in \mathbb{R}_{+}^{2}$. In other words, it holds that $G(\boldsymbol{x}, \boldsymbol{y})=G(\boldsymbol{y}, \boldsymbol{x})^{T}$.

\subsection{The full-plane term}

To calculate $G^{P}(\boldsymbol{x}, \boldsymbol{y})$, it suffices to compute the inverse Fourier transforms of the next three functions (see $(2.14)$ and $(2.39))$ :

$$
\begin{aligned}
& \psi_{1}\left(s, x_{2}\right)=\frac{s^{2} \mathrm{e}^{-\omega \sqrt{s^{2}-s_{0}^{2}}\left|x_{2}-y_{2}\right|}}{\sqrt{s^{2}-s_{0}^{2}}}, \\
& \psi_{2}\left(s, x_{2}\right)=\sqrt{s^{2}-s_{0}^{2}} \mathrm{e}^{-\omega \sqrt{s^{2}-s_{0}^{2}}\left|x_{2}-y_{2}\right|}, \\
& \psi_{3}\left(s, x_{2}\right)=\mathrm{i} \operatorname{sign}\left(x_{2}-y_{2}\right) s \mathrm{e}^{-\omega \sqrt{s^{2}-s_{0}^{2}}\left|x_{2}-y_{2}\right|} \text {, }
\end{aligned}
$$

where $s_{0}>0$ is given. In the calculations, the following integral formulas are required (cf. [1]):

$$
\begin{aligned}
\int_{0}^{\infty} \frac{\mathrm{e}^{-b \sqrt{\xi^{2}+a^{2}}}}{\sqrt{\xi^{2}+a^{2}}} \cos (c \xi) \mathrm{d} \xi & =K_{0}\left(a \sqrt{b^{2}+c^{2}}\right) \\
\int_{0}^{\infty} \mathrm{e}^{-b \sqrt{\xi^{2}+a^{2}}} \cos (c \xi) \mathrm{d} \xi & =\frac{a b}{\sqrt{b^{2}+c^{2}}} K_{1}\left(a \sqrt{b^{2}+c^{2}}\right), \\
\int_{0}^{\infty} \frac{\xi \mathrm{e}^{-b \sqrt{\xi^{2}+a^{2}}}}{\sqrt{\xi^{2}+a^{2}}} \sin (c \xi) \mathrm{d} \xi & =\frac{a c}{\sqrt{b^{2}+c^{2}}} K_{1}\left(a \sqrt{b^{2}+c^{2}}\right), \\
\int_{0}^{\infty} \xi \mathrm{e}^{-b \sqrt{\xi^{2}+a^{2}}} \sin (c \xi) \mathrm{d} \xi & =\frac{a^{2} b c}{b^{2}+c^{2}} K_{2}\left(a \sqrt{b^{2}+c^{2}}\right),
\end{aligned}
$$

where $K_{0}(\cdot), K_{1}(\cdot)$ and $K_{2}(\cdot)$ denote the modified Bessel functions of the second kind of order 0,1 and 2 , respectively (see [2] for their definition and properties). These functions can be expressed in terms of the Hankel functions of the first kind as follows ( $f f$. [2]):

$$
K_{n}(s)=\frac{\pi}{2} \mathrm{i}^{n+1} H_{n}^{(1)}(\mathrm{i} s),
$$


where $s \in \mathbb{C}$ and $n \in \mathbb{N}$. Additionally, we resort to the next recurrence relations for Hankel functions ( $c f$. [2]):

$$
\begin{aligned}
& \frac{\mathrm{d}}{\mathrm{d} s} H_{n}^{(1)}(s)=\frac{n}{s} H_{n}^{(1)}(s)-H_{n+1}^{(1)}(s), \\
& \frac{2 n}{s} H_{n}^{(1)}(s)=H_{n+1}^{(1)}(s)+H_{n-1}^{(1)}(s) .
\end{aligned}
$$

The inverse Fourier transform of (3.3c) is computed by employing formulas (3.4), identity (3.5), and relations (3.6), obtaining

$$
\begin{aligned}
& \mathcal{F}_{\omega}^{-1} \psi_{1}\left(x_{1}, x_{2}\right)=\frac{\mathrm{i} \omega}{2} s_{0}^{2}\left(\frac{1}{\omega s_{0} r} H_{1}^{(1)}\left(\omega s_{0} r\right)-H_{2}^{(1)}\left(\omega s_{0} r\right) \frac{\left(x_{1}-y_{1}\right)^{2}}{r^{2}}\right) \\
& \mathcal{F}_{\omega}^{-1} \psi_{2}\left(x_{1}, x_{2}\right)=\frac{\mathrm{i} \omega}{2} s_{0}^{2}\left(-\frac{1}{\omega s_{0} r} H_{1}^{(1)}\left(\omega s_{0} r\right)+H_{2}^{(1)}\left(\omega s_{0} r\right) \frac{\left(x_{2}-y_{2}\right)^{2}}{r^{2}}\right) \\
& \mathcal{F}_{\omega}^{-1} \psi_{3}\left(x_{1}, x_{2}\right)=-\frac{\mathrm{i} \omega}{2} s_{0}^{2} H_{2}^{(1)}\left(\omega s_{0} r\right) \frac{\left(x_{1}-y_{1}\right)\left(x_{2}-y_{2}\right)}{r^{2}}
\end{aligned}
$$

where $r$ denotes the distance between $\boldsymbol{x}$ and $\boldsymbol{y}$, that is,

$$
r=|\boldsymbol{x}-\boldsymbol{y}|=\sqrt{\left(x_{1}-y_{1}\right)^{2}+\left(x_{2}-y_{2}\right)^{2}} .
$$

Using formulas (3.7) with $s_{0}$ substituted by $s_{L}$ or $s_{T}$ as appropriate, we obtain the following expression for the components of $G^{P}(\boldsymbol{x}, \boldsymbol{y})$ :

$$
G_{i j}^{P}(\boldsymbol{x}, \boldsymbol{y})=\frac{\mathrm{i}}{4 \mu}\left(a(r) \delta_{i j}+b(r) \frac{\left(x_{i}-y_{i}\right)\left(x_{j}-y_{j}\right)}{r^{2}}\right), \quad 1 \leq i, j \leq 2,
$$

where $a(\cdot), b(\cdot)$ are the functions given by

$$
\begin{aligned}
& a(r)=H_{0}^{(1)}\left(\omega s_{T} r\right)-\frac{1}{\omega s_{T} r}\left(H_{1}^{(1)}\left(\omega s_{T} r\right)-\beta H_{1}^{(1)}\left(\omega s_{L} r\right)\right), \\
& b(r)=H_{2}^{(1)}\left(\omega s_{T} r\right)-\beta^{2} H_{2}^{(1)}\left(\omega s_{L} r\right),
\end{aligned}
$$

and $\beta=s_{L} / s_{T}$. The expression (3.9) corresponds exactly to the full-plane Green's function of the timeharmonic elasticity system (see $[3,7]$ for details).

\subsection{The boundary term}

The boundary term $G^{B}(\boldsymbol{x}, \boldsymbol{y})$ requires special attention, because $\widehat{G}^{B}$ has singularities (pseudo-poles and poles) that make difficult the calculation of its inverse Fourier transform. For that reason, these singularities are previously removed by subtracting certain suitable terms, whose inverse transforms are analytically calculable. The advantage of this approach is that the remaining term is regular and its inverse Fourier transform can be numerically approximated. Hence, this procedure decomposes the term $\widehat{G}^{B}$ into a sum of three terms:

$$
\widehat{G}^{B}\left(s, x_{2}\right)=\widehat{G}^{B, \mathrm{reg}}\left(s, x_{2}\right)+\widehat{G}^{B, \mathrm{psp}}\left(s, x_{2}\right)+\widehat{G}^{B, \mathrm{pol}}\left(s, x_{2}\right),
$$

where $\widehat{G}^{B \text {,reg }}$ corresponds to the regular part, $\widehat{G}^{B \text {,psp }}$ is the part of pseudo-poles and $\widehat{G}^{B \text {,pol }}$ is the part of poles. Their inverse Fourier transforms are denoted by $G^{B, \text { reg }}(\boldsymbol{x}, \boldsymbol{y}), G^{B, \mathrm{psp}}(\boldsymbol{x}, \boldsymbol{y})$ and $G^{B, \mathrm{pol}}(\boldsymbol{x}, \boldsymbol{y})$, respectively, and they are separately computed as described below. 


\subsubsection{Pseudo-poles}

Singularities known as pseudo-poles (or half-order poles) are present in the diagonal components $\widehat{G}_{11}^{B}$ and $\widehat{G}_{22}^{B}$, due to the factors $\theta_{L}(s)^{-1}$ and $\theta_{T}(s)^{-1}$, respectively. Specifically, $\widehat{G}_{11}^{B}$ has two pseudo-poles at $s= \pm s_{L}$, whereas $\widehat{G}_{22}^{B}$ has two pseudo-poles at $s= \pm s_{T}$. These singularities can be removed by subtracting the terms

$$
\begin{aligned}
& \widehat{G}_{11}^{B, \operatorname{psp}}\left(s, x_{2}\right)=-\frac{1}{2 \rho \omega} s^{2} \theta_{L}(s)^{-1} \mathrm{e}^{-\omega \theta_{L}(s)\left(x_{2}+y_{2}\right)}, \\
& \widehat{G}_{22}^{B, \operatorname{psp}}\left(s, x_{2}\right)=-\frac{1}{2 \rho \omega} s^{2} \theta_{T}(s)^{-1} \mathrm{e}^{-\omega \theta_{T}(s)\left(x_{2}+y_{2}\right)} .
\end{aligned}
$$

As components $\widehat{G}_{21}^{B}$ and $\widehat{G}_{12}^{B}$ have no pseudo-poles, the term $\widehat{G}^{B \text {,psp }}$ in (3.11) corresponds to a diagonal matrix. Once the subtraction is done, the term $\widehat{G}^{B}-\widehat{G}^{B \text {,psp }}$ can be written as follows:

$$
\left(\widehat{G}^{B}-\widehat{G}^{B, \mathrm{psp}}\right)\left(s, x_{2}\right)=-\frac{1}{2 \rho \omega}\left(\left(2 s^{2}-s_{T}^{2}\right)^{2}-4 s^{2} \theta_{L}(s) \theta_{T}(s)+\mu^{-1} s_{T}^{2} \theta_{T}(s) Z\right)^{-1} \chi\left(s, x_{2}\right),
$$

where $\chi\left(s, x_{2}\right)$ is a $2 \times 2$ matrix function whose components are

$$
\begin{aligned}
\chi_{11}\left(s, x_{2}\right)= & \theta_{T}(s)\left(8 s^{4} \mathrm{e}^{-\omega \theta_{L}(s)\left(x_{2}+y_{2}\right)}\right. \\
& +\left(\left(2 s^{2}-s_{T}^{2}\right)^{2}+4 s^{2} \theta_{L}(s) \theta_{T}(s)-\mu^{-1} s_{T}^{2} \theta_{T}(s) Z\right) \mathrm{e}^{-\omega \theta_{T}(s)\left(x_{2}+y_{2}\right)} \\
& \left.-4 s^{2}\left(2 s^{2}-s_{T}^{2}\right)\left(\mathrm{e}^{-\omega\left(\theta_{L}(s) x_{2}+\theta_{T}(s) y_{2}\right)}+\mathrm{e}^{-\omega\left(\theta_{T}(s) x_{2}+\theta_{L}(s) y_{2}\right)}\right)\right), \\
\chi_{21}\left(s, x_{2}\right)=\mathrm{i} & \left(\left(\left(2 s^{2}-s_{T}^{2}\right)^{2}+4 s^{2} \theta_{L}(s) \theta_{T}(s)+\mu^{-1} s_{T}^{2} \theta_{T}(s) Z\right) \mathrm{e}^{-\omega \theta_{L}(s)\left(x_{2}+y_{2}\right)}\right. \\
& +\left(\left(2 s^{2}-s_{T}^{2}\right)^{2}+4 s^{2} \theta_{L}(s) \theta_{T}(s)-\mu^{-1} s_{T}^{2} \theta_{T}(s) Z\right) \mathrm{e}^{-\omega \theta_{T}(s)\left(x_{2}+y_{2}\right)} \\
& \left.-4\left(2 s^{2}-s_{T}^{2}\right)\left(\theta_{L}(s) \theta_{T}(s) \mathrm{e}^{-\omega\left(\theta_{L}(s) x_{2}+\theta_{T}(s) y_{2}\right)}+s^{2} \mathrm{e}^{-\omega\left(\theta_{T}(s) x_{2}+\theta_{L}(s) y_{2}\right)}\right)\right), \\
\chi_{12}\left(s, x_{2}\right)= & -\mathrm{i} s\left(\left(\left(2 s^{2}-s_{T}^{2}\right)^{2}+4 s^{2} \theta_{L}(s) \theta_{T}(s)+\mu^{-1} s_{T}^{2} \theta_{T}(s) Z\right) \mathrm{e}^{-\omega \theta_{L}(s)\left(x_{2}+y_{2}\right)}\right. \\
& +\left(\left(2 s^{2}-s_{T}^{2}\right)^{2}+4 s^{2} \theta_{L}(s) \theta_{T}(s)-\mu^{-1} s_{T}^{2} \theta_{T}(s) Z\right) \mathrm{e}^{-\omega \theta_{T}(s)\left(x_{2}+y_{2}\right)} \\
& \left.-4\left(2 s^{2}-s_{T}^{2}\right)\left(s^{2} \mathrm{e}^{-\omega\left(\theta_{L}(s) x_{2}+\theta_{T}(s) y_{2}\right)}+\theta_{L}(s) \theta_{T}(s) \mathrm{e}^{-\omega\left(\theta_{T}(s) x_{2}+\theta_{L}(s) y_{2}\right)}\right)\right), \\
\chi_{22}\left(s, x_{2}\right)= & \theta_{L}(s)\left(\left(2 s^{2}-s_{T}^{2}\right)^{2}+4 s^{2} \theta_{L}(s) \theta_{T}(s)+\mu^{-1} s_{T}^{2} \theta_{T}(s) Z\right) \mathrm{e}^{-\omega \theta_{L}(s)\left(x_{2}+y_{2}\right)} \\
& +2 s^{2}\left(4 s^{2} \theta_{L}(s)-\mu^{-1} s_{T}^{2} Z\right) \mathrm{e}^{-\omega \theta_{T}(s)\left(x_{2}+y_{2}\right)} \\
& -4 s^{2}\left(2 s^{2}-s_{T}^{2}\right) \theta_{L}(s)\left(\mathrm{e}^{-\omega\left(\theta_{L}(s) x_{2}+\theta_{T}(s) y_{2}\right)}+\mathrm{e}^{-\omega\left(\theta_{T}(s) x_{2}+\theta_{L}(s) y_{2}\right)}\right) .
\end{aligned}
$$

These functions do not have any singularity. Moreover, it is straightforward to verify that $\chi_{11}, \chi_{22}$ are even functions in $s$, and $\chi_{21}, \chi_{12}$ are odd functions in $s$. Then, the inverse Fourier transform of (3.12) is analytically computed by employing formulas (3.4), (3.5), and (3.8), obtaining

$$
\begin{aligned}
& G_{11}^{B, \operatorname{psp}}(\boldsymbol{x}, \boldsymbol{y})=-\frac{\mathrm{i} \beta^{2}}{4 \mu}\left(\frac{1}{\omega s_{L} \bar{r}} H_{1}^{(1)}\left(\omega s_{L} \bar{r}\right)-H_{2}^{(1)}\left(\omega s_{L} \bar{r}\right) \frac{\left(x_{1}-y_{1}\right)^{2}}{\bar{r}^{2}}\right) \\
& G_{22}^{B, \operatorname{psp}}(\boldsymbol{x}, \boldsymbol{y})=-\frac{\mathrm{i}}{4 \mu}\left(\frac{1}{\omega s_{T} \bar{r}} H_{1}^{(1)}\left(\omega s_{T} \bar{r}\right)-H_{2}^{(1)}\left(\omega s_{T} \bar{r}\right) \frac{\left(x_{1}-y_{1}\right)^{2}}{\bar{r}^{2}}\right)
\end{aligned}
$$

where

$$
\bar{r}=\sqrt{\left(x_{1}-y_{1}\right)^{2}+\left(x_{2}+y_{2}\right)^{2}} .
$$




\subsubsection{Poles}

The poles of the spectral Green's function are removed from the term $\widehat{G}^{B}-\widehat{G}^{B \text {,psp }}$ given by (3.13), which does not have pseudo-poles. As the matrix function $\chi\left(s, x_{2}\right)$ is regular in $s$, all the poles come from the common denominator in (3.13). These poles are located at those values of $s$ such that this denominator vanishes, that is, they are solutions to the equation

$$
\left(2 s^{2}-s_{T}^{2}\right)^{2}-4 s^{2} \theta_{L}(s) \theta_{T}(s)+\mu^{-1} s_{T}^{2} \theta_{T}(s) Z=0 .
$$

This equation can not, in general, be solved analytically. So it is necessary to employ numerical procedures, and this matter is discussed in the next section. Nevertheless, as (3.17) only depends on $s^{2}$ (see (2.14)), we can establish a priori that the poles correspond to pairs symmetrically located with respect to the origin in the complex plane. Thus, we take two solutions, namely, $s=\hat{s}$ and $s=-\hat{s}$, with $\mathfrak{R e}(\hat{s})>0$, and we assume that $\widehat{G}^{B}-\widehat{G}^{B \text {,psp }}$ has two simple poles at these locations. The method we use to extract the poles is based on subtracting a simple term from $\widehat{G}^{B}-\widehat{G}^{B}$,psp, which behaves in the same way at the neighborhood of each pole and is regular elsewhere. We express this term as follows:

$$
\widehat{G}^{B, \operatorname{pol}}\left(s, x_{2}\right)=-\frac{1}{2 \rho \omega}\left(\frac{1}{s-\hat{s}} C^{+}\left(\hat{s}, x_{2}\right)+\frac{1}{s+\hat{s}} C^{-}\left(\hat{s}, x_{2}\right)\right),
$$

where $C^{+}\left(\hat{s}, x_{2}\right)$ and $C^{-}\left(\hat{s}, x_{2}\right)$ are the residue matrices of the poles at $s=\hat{s}$ and $s=-\hat{s}$, respectively. These matrices correspond to the next limits

$$
\begin{aligned}
& C^{+}\left(\hat{s}, x_{2}\right)=-2 \rho \omega \lim _{s \rightarrow+\hat{s}}(s-\hat{s})\left(\widehat{G}^{B}-\widehat{G}^{B, \mathrm{psp}}\right)\left(s, x_{2}\right), \\
& C^{-}\left(\hat{s}, x_{2}\right)=-2 \rho \omega \lim _{s \rightarrow-\hat{s}}(s+\hat{s})\left(\widehat{G}^{B}-\widehat{G}^{B, \mathrm{psp}}\right)\left(s, x_{2}\right) .
\end{aligned}
$$

Combining with (3.13), we obtain

$$
\begin{aligned}
C^{+}\left(\hat{s}, x_{2}\right) & =\lim _{s \rightarrow+\hat{s}} \frac{(s-\hat{s}) \chi\left(s, x_{2}\right)}{\left(2 s^{2}-s_{T}^{2}\right)^{2}-4 s^{2} \theta_{L}(s) \theta_{T}(s)+\mu^{-1} s_{T}^{2} \theta_{T}(s) Z} \\
C^{-}\left(\hat{s}, x_{2}\right) & =\lim _{s \rightarrow-\hat{s}} \frac{(s+\hat{s}) \chi\left(s, x_{2}\right)}{\left(2 s^{2}-s_{T}^{2}\right)^{2}-4 s^{2} \theta_{L}(s) \theta_{T}(s)+\mu^{-1} s_{T}^{2} \theta_{T}(s) Z} .
\end{aligned}
$$

Because of the evenness or oddness of each component of $\chi\left(s, x_{2}\right)$ (see (3.14)), it is direct to verify that the next symmetries hold:

$$
\begin{array}{ll}
C_{11}^{+}\left(\hat{s}, x_{2}\right)=-C_{11}^{-}\left(\hat{s}, x_{2}\right), & C_{12}^{+}\left(\hat{s}, x_{2}\right)=C_{12}^{-}\left(\hat{s}, x_{2}\right), \\
C_{21}^{+}\left(\hat{s}, x_{2}\right)=C_{21}^{-}\left(\hat{s}, x_{2}\right), & C_{22}^{+}\left(\hat{s}, x_{2}\right)=-C_{22}^{-}\left(\hat{s}, x_{2}\right) .
\end{array}
$$

Hence, to simplify notation, we put $c_{i j}\left(\hat{s}, x_{2}\right)=C_{i j}^{+}\left(\hat{s}, x_{2}\right)$, and the matrices $C^{+}\left(\hat{s}, x_{2}\right)$ and $C^{-}\left(\hat{s}, x_{2}\right)$ are expressed as follows:

$$
C_{+}\left(\hat{s}, x_{2}\right)=\left[\begin{array}{ll}
c_{11}\left(\hat{s}, x_{2}\right) & c_{12}\left(\hat{s}, x_{2}\right) \\
c_{21}\left(\hat{s}, x_{2}\right) & c_{22}\left(\hat{s}, x_{2}\right)
\end{array}\right], \quad C_{-}\left(\hat{s}, x_{2}\right)=\left[\begin{array}{rr}
-c_{11}\left(\hat{s}, x_{2}\right) & c_{12}\left(\hat{s}, x_{2}\right) \\
c_{21}\left(\hat{s}, x_{2}\right) & -c_{22}\left(\hat{s}, x_{2}\right)
\end{array}\right]
$$

where the coefficients $c_{i j}\left(\hat{s}, x_{2}\right)$ can be computed by using L'Hôpital's rule:

$$
c_{i j}\left(\hat{s}, x_{2}\right)=\frac{\chi_{i j}\left(\hat{s}, x_{2}\right)}{\left.\frac{\mathrm{d}}{\mathrm{d} s}\left(\left(2 s^{2}-s_{T}^{2}\right)^{2}-4 s^{2} \theta_{L}(s) \theta_{T}(s)+\mu^{-1} s_{T}^{2} \theta_{T}(s) Z\right)\right|_{s=\hat{s}}}
$$


(a)

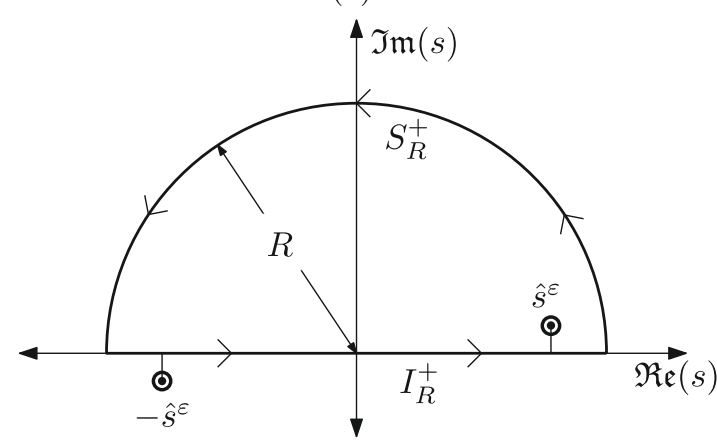

(b)

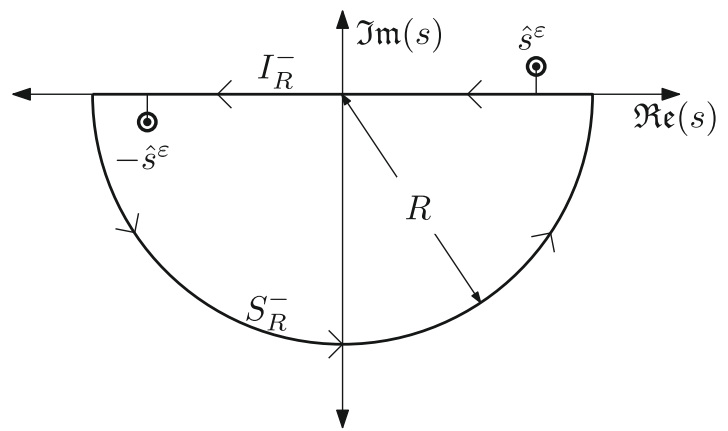

Figure 2. Contours in the complex plane for cases (a) $x_{1}-y_{1} \geq 0$ and (b) $x_{1}-y_{1} \leq 0$.

The inverse Fourier transform of (3.18) is analytically computed. In order to determine the diagonal terms of $G^{B}-G^{B, p s p}$, the next inverse transform has to be calculated:

$$
\mathcal{F}_{\omega}^{-1}\left(\frac{1}{s-\hat{s}}-\frac{1}{s+\hat{s}}\right)(\boldsymbol{x}, \boldsymbol{y})=\frac{\omega}{2 \pi} \int_{-\infty}^{+\infty}\left(\frac{1}{s-\hat{s}}-\frac{1}{s+\hat{s}}\right) \mathrm{e}^{\mathrm{i} \omega s\left(x_{1}-y_{1}\right)} \mathrm{d} s .
$$

Let us assume that $\hat{s}$ is strictly real. In that case, the computation of the integral in (3.24) must be carefully made, because the functions $1 /(s-\hat{s})$ and $1 /(s+\hat{s})$ are not integrable in $\mathbb{R}$. In order to overcome this difficulty, the pole at $s=\hat{s}$ is regarded as the limit

$$
\hat{s}=\lim _{\varepsilon \rightarrow 0^{+}} \hat{s}^{\varepsilon}=\lim _{\varepsilon \rightarrow 0^{+}} \hat{s}+\mathrm{i} \varepsilon
$$

Therefore, the calculation is made assuming that the poles are placed at $s= \pm \hat{s}^{\varepsilon}$, where $\varepsilon>0$ is fixed. This procedure takes out both poles from the real axis and the inverse Fourier transform becomes correctly defined in the classical sense. Notice that the pole at $s=\hat{s}$ is displaced to the upper complex half-plane, whereas the pole at $s=-\hat{s}$ is displaced to the lower complex half-plane. This particular choice of sign for the imaginary part of each pole gives the solution having the right physical sense, that is, outgoing waves (for a more detailed explanation, see $[9,10])$. Once the inverse transform has been computed, we take the limit $\varepsilon \longrightarrow 0^{+}$at the expression we have obtained. Let us define the function $f_{\varepsilon}=f_{\varepsilon}(s)$ as the term under the integral sign in (3.24) when $\hat{s}$ is replaced by $\hat{s}^{\varepsilon}$, that is,

$$
f_{\varepsilon}(s)=\left(\frac{1}{s-\hat{s}^{\varepsilon}}-\frac{1}{s+\hat{s}^{\varepsilon}}\right) \mathrm{e}^{\mathrm{i} \omega s\left(x_{1}-y_{1}\right)}
$$

This function has two complex simple poles at $s=\hat{s}^{\varepsilon}$ and at $s=-\hat{s}^{\varepsilon}$. The integral in (3.24) is computed by application of Cauchy's residues theorem. This calculation is separately performed for the cases $x_{1}-y_{1} \geq 0$ and $x_{1}-y_{1} \leq 0$, dealing with different clockwise-oriented contours in the complex plane for each case. These contours are shown in Figure 2, where $R>\left|\hat{s}^{\varepsilon}\right|$ is a parameter destined to tend to infinity. When $x_{1}-y_{1} \geq 0$, we integrate along the upper contour, which consists of two parts, namely, the straight line $I_{R}^{+}$and the upper semicircle $S_{R}^{+}$(see Fig. 2a). Notice that only the pole at $s=\hat{s}^{\varepsilon}$ lies inside this contour, then it follows from the residues theorem that

$$
\int_{I_{R}^{+}} f_{\varepsilon}(s) \mathrm{d} s+\int_{S_{R}^{+}} f_{\varepsilon}(s) \mathrm{d} s=2 \pi \mathrm{i} \underset{s=\hat{s}^{\varepsilon}}{\operatorname{Res}} f_{\varepsilon}(s)
$$


As the contour is clockwise oriented, the following limit is immediate:

$$
\int_{I_{R}^{+}} f_{\varepsilon}(s) \mathrm{d} s \underset{R \longrightarrow+\infty}{\longrightarrow} \int_{-\infty}^{+\infty}\left(\frac{1}{s-\hat{s}^{\varepsilon}}-\frac{1}{s+\hat{s}^{\varepsilon}}\right) \mathrm{e}^{\mathrm{i} \omega s\left(x_{1}-y_{1}\right)} \mathrm{d} s .
$$

Moreover, it is straightforward to prove the estimation

$$
\left|\int_{S_{R}^{+}} f_{\varepsilon}(s) \mathrm{d} s\right| \leq \int_{0}^{\pi} \frac{2 R\left|\hat{s}^{\varepsilon}\right|}{R^{2}-\left|\hat{s}^{\varepsilon}\right|^{2}} \mathrm{e}^{-\omega R \sin \theta\left(x_{1}-y_{1}\right)} \mathrm{d} \theta
$$

and as $x_{1}-y_{1} \geq 0$, it follows from (3.29) that

$$
\int_{S_{R}^{+}} f_{\varepsilon}(s) \mathrm{d} s \underset{R \longrightarrow+\infty}{\longrightarrow} 0 .
$$

On the other hand, the residue of $f_{\varepsilon}$ associated with the pole at $s=\hat{s}^{\varepsilon}$ is computed as follows:

$$
\operatorname{Res}_{s=\hat{s}^{\varepsilon}} f_{\varepsilon}(s)=\lim _{s \rightarrow \hat{s}^{\varepsilon}}\left(s-\hat{s}^{\varepsilon}\right) f_{\varepsilon}(s)=\lim _{s \rightarrow \hat{s}^{\varepsilon}}\left(1-\frac{s-\hat{s}^{\varepsilon}}{s+\hat{s}^{\varepsilon}}\right) \mathrm{e}^{\mathrm{i} \omega s\left(x_{1}-y_{1}\right)}=\mathrm{e}^{\mathrm{i} \omega \hat{s}^{\varepsilon}\left(x_{1}-y_{1}\right)} .
$$

Consequently, taking the limit $R \longrightarrow+\infty$ in (3.27) and replacing (3.28), (3.30), and (3.31) leads to the identity

$$
\int_{-\infty}^{+\infty}\left(\frac{1}{s-\hat{s}^{\varepsilon}}-\frac{1}{s+\hat{s}^{\varepsilon}}\right) \mathrm{e}^{\mathrm{i} \omega s\left(x_{1}-y_{1}\right)} \mathrm{d} s=2 \pi \mathrm{i} \mathrm{e}^{\mathrm{i} \omega \hat{s}^{\varepsilon}\left(x_{1}-y_{1}\right)}
$$

which is valid when $x_{1}-y_{1} \geq 0$. In the case $x_{1}-y_{1} \leq 0$, we integrate along the lower contour, which consists of the straight line $I_{R}^{-}$and the lower semicircle $S_{R}^{-}$(see Fig. 2b). The procedure is analogous to the previous case, but this time it is necessary to take into account that this contour only encircles the pole at $s=-\hat{s}^{\varepsilon}$. We finally obtain the identity

$$
\int_{-\infty}^{+\infty}\left(\frac{1}{s-\hat{s}^{\varepsilon}}-\frac{1}{s+\hat{s}^{\varepsilon}}\right) \mathrm{e}^{\mathrm{i} \omega s\left(x_{1}-y_{1}\right)} \mathrm{d} s=2 \pi \mathrm{ie}^{-\mathrm{i} \omega \hat{s}^{\varepsilon}\left(x_{1}-y_{1}\right)}
$$

which is valid when $x_{1}-y_{1} \leq 0$. In the general case, both identities (3.32) and (3.33) can be expressed in a single form:

$$
\int_{-\infty}^{+\infty}\left(\frac{1}{s-\hat{s}^{\varepsilon}}-\frac{1}{s+\hat{s}^{\varepsilon}}\right) \mathrm{e}^{\mathrm{i} \omega s\left(x_{1}-y_{1}\right)} \mathrm{d} s=2 \pi \mathrm{i} \mathrm{e}^{\mathrm{i} \omega \hat{s}^{\varepsilon}\left|x_{1}-y_{1}\right|} .
$$

Finally, taking the limit $\varepsilon \longrightarrow 0^{+}$in (3.34) and replacing in (3.24), we obtain

$$
\mathcal{F}_{\omega}^{-1}\left(\frac{1}{s-\hat{s}}-\frac{1}{s+\hat{s}}\right)(\boldsymbol{x}, \boldsymbol{y})=\mathrm{i} \omega \mathrm{e}^{\mathrm{i} \omega \hat{s}\left|x_{1}-y_{1}\right|}
$$

In order to determine the off-diagonal terms of $G^{B}-G^{B, p s p}$, it is necessary to compute:

$$
\mathcal{F}_{\omega}^{-1}\left(\frac{1}{s-\hat{s}}+\frac{1}{s+\hat{s}}\right)(\boldsymbol{x}, \boldsymbol{y})=\frac{\omega}{2 \pi} \int_{-\infty}^{+\infty}\left(\frac{1}{s-\hat{s}}+\frac{1}{s+\hat{s}}\right) \mathrm{e}^{\mathrm{i} \omega s\left(x_{1}-y_{1}\right)} \mathrm{d} s .
$$

The development for calculating the integral in (3.36) is completely analogous to that already done for (3.24). The final result is

$$
\mathcal{F}_{\omega}^{-1}\left(\frac{1}{s-\hat{s}}+\frac{1}{s+\hat{s}}\right)(\boldsymbol{x}, \boldsymbol{y})=\mathrm{i} \omega \operatorname{sign}\left(x_{1}-y_{1}\right) \mathrm{e}^{i \omega \hat{s}\left|x_{1}-y_{1}\right|} .
$$


If $\hat{s}$ has a nonzero imaginary part, the inverse Fourier transform of (3.18) can be computed directly by using the Cauchy's residues theorem, leading to the same result in function of $\hat{s}$. Then, substituting (3.35) and (3.37) in (3.18) yields the components of $G^{B, \text { pol }}$ :

$$
\begin{aligned}
& G_{11}^{B, \text { pol }}(\boldsymbol{x}, \boldsymbol{y})=-\frac{\mathrm{i}}{2 \rho} c_{11}\left(\hat{s}, x_{2}\right) \mathrm{e}^{\mathrm{i} \omega \hat{s}\left|x_{1}-y_{1}\right|}, \\
& G_{21}^{B, \mathrm{pol}}(\boldsymbol{x}, \boldsymbol{y})=-\frac{\mathrm{i}}{2 \rho} c_{21}\left(\hat{s}, x_{2}\right) \operatorname{sign}\left(x_{1}-y_{1}\right) \mathrm{e}^{\mathrm{i} \omega \hat{s}\left|x_{1}-y_{1}\right|}, \\
& G_{12}^{B, \text { pol }}(\boldsymbol{x}, \boldsymbol{y})=-\frac{\mathrm{i}}{2 \rho} c_{12}\left(\hat{s}, x_{2}\right) \operatorname{sign}\left(x_{1}-y_{1}\right) \mathrm{e}^{\mathrm{i} \omega \hat{s}\left|x_{1}-y_{1}\right|}, \\
& G_{22}^{B, \text { pol }}(\boldsymbol{x}, \boldsymbol{y})=-\frac{\mathrm{i}}{2 \rho} c_{22}\left(\hat{s}, x_{2}\right) \mathrm{e}^{\mathrm{i} \omega \hat{s}\left|x_{1}-y_{1}\right|},
\end{aligned}
$$

where explicit expression for the coefficients $c_{i j}$ can be obtained by replacing (3.14) in (3.23) and expanding:

$$
\begin{aligned}
& c_{11}\left(\hat{s}, x_{2}\right)=\frac{\theta_{T}(\hat{s})}{\hat{s}}\left(4\left(2 \hat{s}^{2}-s_{T}^{2}\right)-8 \theta_{L}(\hat{s}) \theta_{T}(\hat{s})-4 \hat{s}^{2}\left(\frac{\theta_{L}(\hat{s})}{\theta_{T}(\hat{s})}+\frac{\theta_{T}(\hat{s})}{\theta_{L}(\hat{s})}\right)+\mu^{-1} \frac{s_{T}^{2}}{\theta_{T}(\hat{s})} Z\right)^{-1} \\
& \times\left(8 \hat{s}^{4} \mathrm{e}^{-\omega \theta_{L}(\hat{s})\left(x_{2}+y_{2}\right)}+\left(\left(2 \hat{s}^{2}-s_{T}^{2}\right)^{2}+4 \hat{s}^{2} \theta_{L}(\hat{s}) \theta_{T}(\hat{s})-\mu^{-1} s_{T}^{2} \theta_{T}(\hat{s}) Z\right) \mathrm{e}^{-\omega \theta_{T}(\hat{s})\left(x_{2}+y_{2}\right)}\right. \\
& \left.-4 \hat{s}^{2}\left(2 \hat{s}^{2}-s_{T}^{2}\right)\left(\mathrm{e}^{-\omega\left(\theta_{L}(\hat{s}) x_{2}+\theta_{T}(\hat{s}) y_{2}\right)}+\mathrm{e}^{-\omega\left(\theta_{T}(\hat{s}) x_{2}+\theta_{L}(\hat{s}) y_{2}\right)}\right)\right), \\
& c_{21}\left(\hat{s}, x_{2}\right)=\mathrm{i}\left(4\left(2 \hat{s}^{2}-s_{T}^{2}\right)-8 \theta_{L}(\hat{s}) \theta_{T}(\hat{s})-4 \hat{s}^{2}\left(\frac{\theta_{L}(\hat{s})}{\theta_{T}(\hat{s})}+\frac{\theta_{T}(\hat{s})}{\theta_{L}(\hat{s})}\right)+\mu^{-1} \frac{s_{T}^{2}}{\theta_{T}(\hat{s})} Z\right)^{-1} \\
& \times\left(\left(\left(2 \hat{s}^{2}-s_{T}^{2}\right)^{2}+4 \hat{s}^{2} \theta_{L}(\hat{s}) \theta_{T}(\hat{s})+\mu^{-1} s_{T}^{2} \theta_{T}(\hat{s}) Z\right) \mathrm{e}^{-\omega \theta_{L}(\hat{s})\left(x_{2}+y_{2}\right)}\right. \\
& +\left(\left(2 \hat{s}^{2}-s_{T}^{2}\right)^{2}+4 \hat{s}^{2} \theta_{L}(\hat{s}) \theta_{T}(\hat{s})-\mu^{-1} s_{T}^{2} \theta_{T}(\hat{s}) Z\right) \mathrm{e}^{-\omega \theta_{T}(\hat{s})\left(x_{2}+y_{2}\right)} \\
& \left.-4\left(2 \hat{s}^{2}-s_{T}^{2}\right)\left(\theta_{L}(\hat{s}) \theta_{T}(\hat{s}) \mathrm{e}^{-\omega\left(\theta_{L}(\hat{s}) x_{2}+\theta_{T}(\hat{s}) y_{2}\right)}+\hat{s}^{2} \mathrm{e}^{-\omega\left(\theta_{T}(\hat{s}) x_{2}+\theta_{L}(\hat{s}) y_{2}\right)}\right)\right), \\
& c_{12}\left(\hat{s}, x_{2}\right)=-\mathrm{i}\left(4\left(2 \hat{s}^{2}-s_{T}^{2}\right)-8 \theta_{L}(\hat{s}) \theta_{T}(\hat{s})-4 \hat{s}^{2}\left(\frac{\theta_{L}(\hat{s})}{\theta_{T}(\hat{s})}+\frac{\theta_{T}(\hat{s})}{\theta_{L}(\hat{s})}\right)+\mu^{-1} \frac{s_{T}^{2}}{\theta_{T}(\hat{s})} Z\right)^{-1} \\
& \times\left(\left(\left(2 \hat{s}^{2}-s_{T}^{2}\right)^{2}+4 \hat{s}^{2} \theta_{L}(\hat{s}) \theta_{T}(\hat{s})+\mu^{-1} s_{T}^{2} \theta_{T}(\hat{s}) Z\right) \mathrm{e}^{-\omega \theta_{L}(\hat{s})\left(x_{2}+y_{2}\right)}\right. \\
& +\left(\left(2 \hat{s}^{2}-s_{T}^{2}\right)^{2}+4 \hat{s}^{2} \theta_{L}(\hat{s}) \theta_{T}(\hat{s})-\mu^{-1} s_{T}^{2} \theta_{T}(\hat{s}) Z\right) \mathrm{e}^{-\omega \theta_{T}(\hat{s})\left(x_{2}+y_{2}\right)} \\
& \left.-4\left(2 \hat{s}^{2}-s_{T}^{2}\right)\left(\hat{s}^{2} \mathrm{e}^{-\omega\left(\theta_{L}(\hat{s}) x_{2}+\theta_{T}(\hat{s}) y_{2}\right)}+\theta_{L}(\hat{s}) \theta_{T}(\hat{s}) \mathrm{e}^{-\omega\left(\theta_{T}(\hat{s}) x_{2}+\theta_{L}(\hat{s}) y_{2}\right)}\right)\right), \\
& c_{22}\left(\hat{s}, x_{2}\right)=\frac{\theta_{L}(\hat{s})}{\hat{s}}\left(4\left(2 \hat{s}^{2}-s_{T}^{2}\right)-8 \theta_{L}(\hat{s}) \theta_{T}(\hat{s})-4 \hat{s}^{2}\left(\frac{\theta_{L}(\hat{s})}{\theta_{T}(\hat{s})}+\frac{\theta_{T}(\hat{s})}{\theta_{L}(\hat{s})}\right)+\mu^{-1} \frac{s_{T}^{2}}{\theta_{T}(\hat{s})} Z\right)^{-1} \\
& \times\left(\left(\left(2 \hat{s}^{2}-s_{T}^{2}\right)^{2}+4 \hat{s}^{2} \theta_{L}(\hat{s}) \theta_{T}(\hat{s})+\mu^{-1} s_{T}^{2} \theta_{T}(\hat{s}) Z\right) \mathrm{e}^{-\omega \theta_{L}(\hat{s})\left(x_{2}+y_{2}\right)}\right. \\
& +2 \hat{s}^{2}\left(4 \hat{s}^{2}-\mu^{-1} s_{T}^{2} \theta_{L}(\hat{s})^{-1} Z\right) \mathrm{e}^{-\omega \theta_{T}(\hat{s})\left(x_{2}+y_{2}\right)} \\
& \left.-4 \hat{s}^{2}\left(2 \hat{s}^{2}-s_{T}^{2}\right)\left(\mathrm{e}^{-\omega\left(\theta_{L}(\hat{s}) x_{2}+\theta_{T}(\hat{s}) y_{2}\right)}+\mathrm{e}^{-\omega\left(\theta_{T}(\hat{s}) x_{2}+\theta_{L}(\hat{s}) y_{2}\right)}\right)\right) .
\end{aligned}
$$

The expressions (3.38) and (3.39) complete that presented in the previous article [8]. 


\subsubsection{Regular part}

Once the terms corresponding to pseudo-poles and poles have been subtracted from $\widehat{G}^{B}$, the following term remains:

$$
\widehat{G}^{B, \mathrm{reg}}\left(s, x_{2}\right)=\widehat{G}^{B}\left(s, x_{2}\right)-\widehat{G}^{B, \mathrm{psp}}\left(s, x_{2}\right)-\widehat{G}^{B, \mathrm{pol}}\left(s, x_{2}\right),
$$

which is regular, because all its singularities in $s$ have been removed. Furthermore, $\widehat{G}^{B}$ decreases fast at infinity in $s$, since $\widehat{G}^{B}-\widehat{G}^{B \text {,psp }}$ is exponentially decreasing. The inverse Fourier transform of $\widehat{G}^{B, \text { reg }}$ is numerically approximated by an inverse discrete Fourier transform (IDFT). In order to perform this computation, we resort to an algorithm of backward Fast Fourier Transform (FFT). Both the spatial and spectral domains are truncated in a symmetric way by considering the intervals $\left[-x_{\max }, x_{\max }\right]$ and $\left[-s_{\max }, s_{\max }\right]$ where $x_{\max }>0$ and $s_{\max }>0$ are maximum values for $\left|x_{1}\right|$ and $|s|$, respectively. These domains are discretized by taking $N$ equispaced samples for both variables. Thus, if $\phi\left(x_{1}\right)$ is a function and $\Phi(s)$ is its Fourier transform $\mathcal{F}_{\omega}$, their discrete approximations $\phi_{n}$ and $\Phi_{m}$, are

$$
\Phi_{m}=\frac{x_{\max }}{N} \sum_{n=0}^{N-1} \phi_{n} \mathrm{e}^{-\frac{2 \pi \mathrm{in} m}{N \omega}}, \quad \phi_{n}=\frac{2 \omega}{\pi} \frac{s_{\max }}{N} \sum_{m=0}^{N-1} \Phi_{m} \mathrm{e}^{\frac{2 \pi \mathrm{in} m}{N \omega}}, \quad 0 \leq n, m \leq N-1 .
$$

This algorithm gives a numerical approximation of $G^{B, \operatorname{reg}}(\boldsymbol{x}, \boldsymbol{y})$ in a bounded region of $\mathbb{R}_{+}^{2}$.

\section{Numerical COMPUtation OF POles}

The poles of the spectral Green's function are determined by solving (3.17). This equation can be expressed in terms of $s_{L}$ and $s_{T}$ by substituting (2.14) in (3.17):

$$
\left(2 s^{2}-s_{T}^{2}\right)^{2}-4 s^{2} \sqrt{s^{2}-s_{L}^{2}} \sqrt{s^{2}-s_{T}^{2}}+\mu^{-1} s_{T}^{2} \sqrt{s^{2}-s_{T}^{2}} Z=0 .
$$

In general, (4.1) can only be solved by numerical methods. Nevertheless, when $Z=0$ an analytical solution is possible. In this case, this equation becomes:

$$
\left(2 s^{2}-s_{T}^{2}\right)^{2}-4 s^{2} \sqrt{s^{2}-s_{L}^{2}} \sqrt{s^{2}-s_{T}^{2}}=0 .
$$

Replacing $s_{L}$ and $s_{T}$ from (2.12) and expanding, we obtain that $s^{2}$ is a root of a third degree equation:

$$
x^{3}+a_{2} x^{2}+a_{1} x+a_{0}=0,
$$

where the coefficients $a_{0}, a_{1}$ and $a_{2}$ are given by

$$
a_{0}=-\frac{s_{T}^{8}}{16\left(s_{T}^{2}-s_{L}^{2}\right)}, \quad a_{1}=\frac{s_{T}^{6}}{2\left(s_{T}^{2}-s_{L}^{2}\right)}, \quad a_{2}=-\frac{s_{T}^{2}\left(3 s_{T}^{2}-2 s_{L}^{2}\right)}{2\left(s_{T}^{2}-s_{L}^{2}\right)} .
$$

This polynomial equation can be analytically solved by means of well-known explicit formulas for their roots. In the general case $Z \in \mathbb{R}$, the roots of (4.1) are numerically approximated by a Newton's algorithm.

Next, we present a numerical example of calculation of poles. The elastic medium we consider is limestone, which is a sedimentary rock whose physical parameters are approximately ( $c f .[19])$ :

$$
\rho=2.400\left[\mathrm{~kg} \cdot \mathrm{m}^{-3}\right], \quad E=70[\mathrm{GPa}], \quad \nu=0.3,
$$

where $E$ is the Young's modulus and $\nu$ is the Poisson's ratio. The Lamé's constants can be obtained by the well-known formulas

$$
\lambda=\frac{\nu E}{(1+\nu)(1-2 \nu)}, \quad \mu=\frac{E}{2(1+\nu)} .
$$




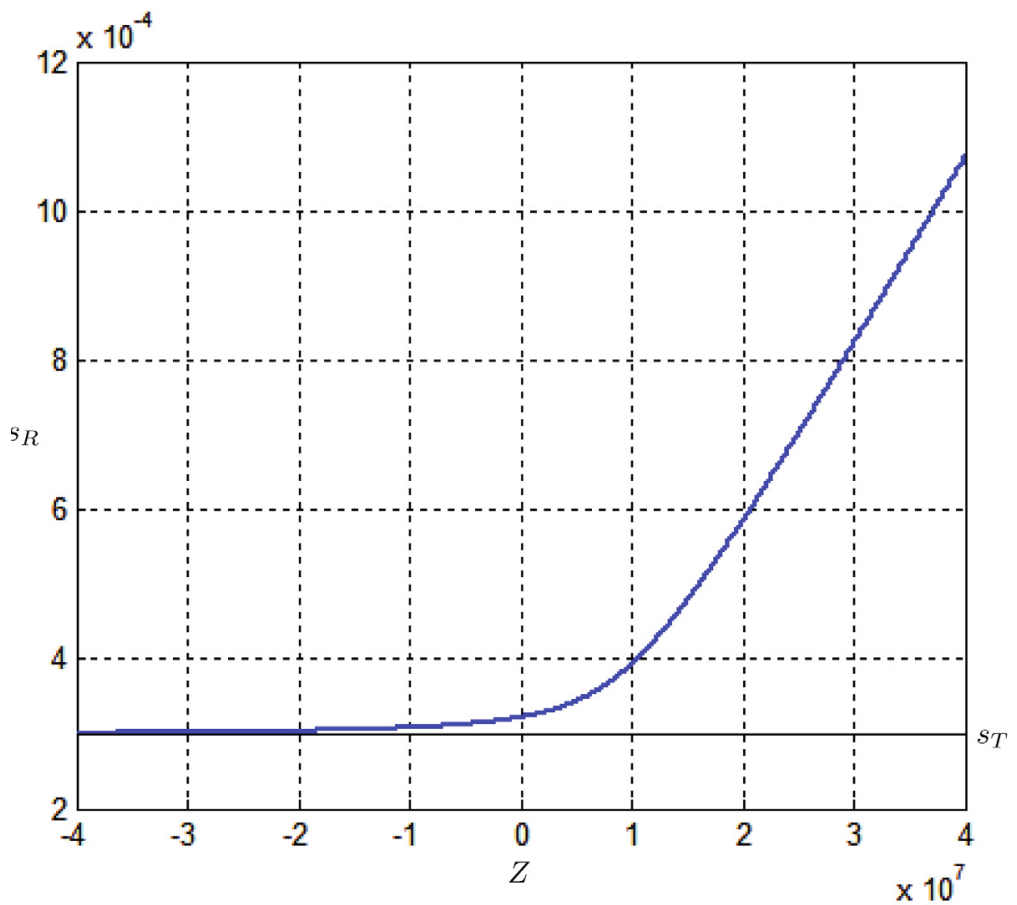

Figure 3. Dependence of Rayleigh slowness with $Z$.

Replacing the values of $E$ and $\nu$ from (4.5) in (4.6) yields the following values for $\lambda$ and $\mu$ :

$$
\lambda=40.3846[\mathrm{GPa}], \quad \mu=26.9231[\mathrm{GPa}]
$$

and replacing these values in formulas (2.12) we obtain the following numerical values for the slownesses:

$$
s_{L}=1.5959 \times 10^{-4}\left[\mathrm{~s} \cdot \mathrm{m}^{-1}\right], \quad s_{T}=2.9857 \times 10^{-4}\left[\mathrm{~s} \cdot \mathrm{m}^{-1}\right] .
$$

Applying the foregoing procedure, we have found that for all $Z$ in $\mathbb{R}$, there exist two real poles at the locations $s=s_{R}$ and $s=-s_{R}$. The positive quantity $s_{R}$ corresponds to the Rayleigh slowness, which is associated with the well-known Rayleigh surface wave, guided by the infinity boundary. Its value varies with the impedance $Z$ as indicated in Figure 3, where we can observe that $s_{R}$ is an increasing function of $Z$, which approaches asymptotically $s_{T}$ when $Z$ decreases to $-\infty$. Moreover, we have found by numerical evidence that for certain values of $Z$, the spectral Green's function has two additional poles, which are in general complexes. These poles can be written as $s= \pm\left(s_{A}+\mathrm{i} \delta_{A}\right)$ where $s_{A}$ and $\delta_{A}$ are positives quantities. The whole curve described by the positive pole as a function of $Z$ in the complex plane is shown in Figure 4, where the zones corresponding to true poles of the spectral Green's function are emphasized. Notice that these poles are located at the zone $s_{L}<\mathfrak{R e}(s)<s_{T}$, this is due to the definition of the complex square roots considered in (4.1) (see Fig. 1). The dependence of $s_{A}$ and $\delta_{A}$ on $Z$ is shown in Figures $5 \mathrm{a}$ and $5 \mathrm{~b}$, respectively, where the solution area is indicated again. Figures 4 and 5 put in evidence that there exists a positive value of the impedance, which we denote by $Z^{*}$, such that the imaginary part $\delta_{A}$ vanishes. In this particular case, $\widehat{G}$ has two additional real poles and there appears another surface wave, whose slowness is denoted by $s^{*}$. Indeed, the exact values of $Z^{*}$ and $s^{*}$ can 


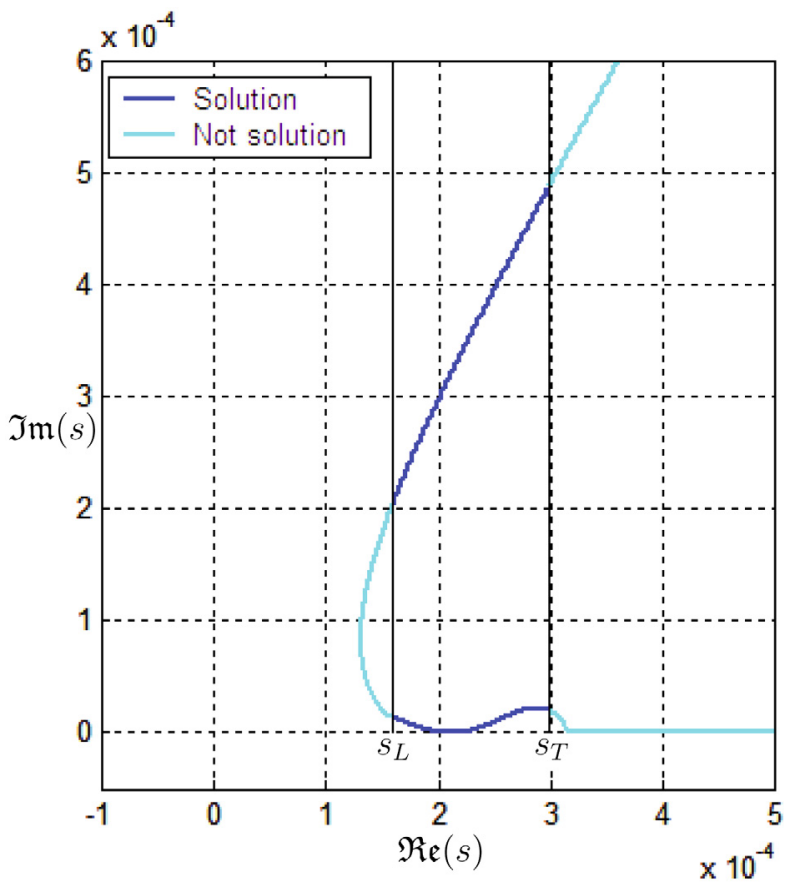

Figure 4. Additional pole behavior in the complex plane as a function of $Z$.
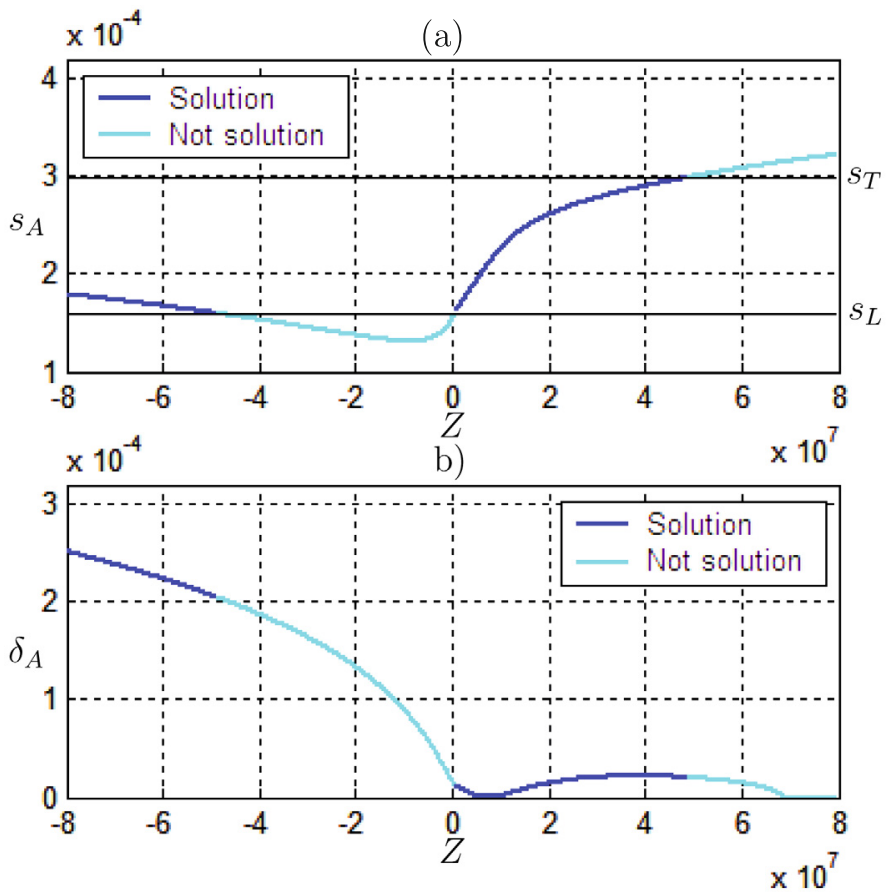

Figure 5. Additional pole as a function of $Z$. (a) Real part $s_{A}$. (b) Imaginary part $\delta_{A}$. 
(a)

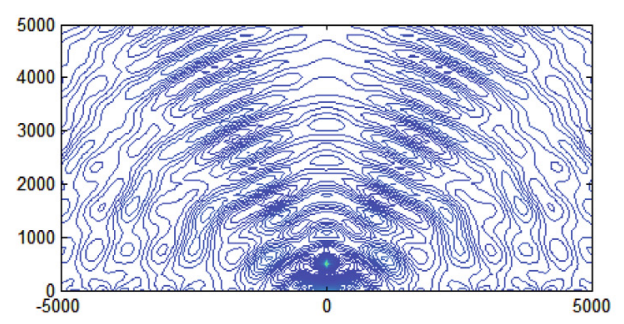

(c)

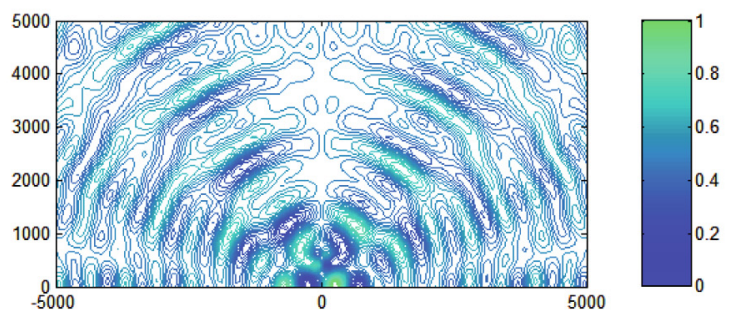

(b)

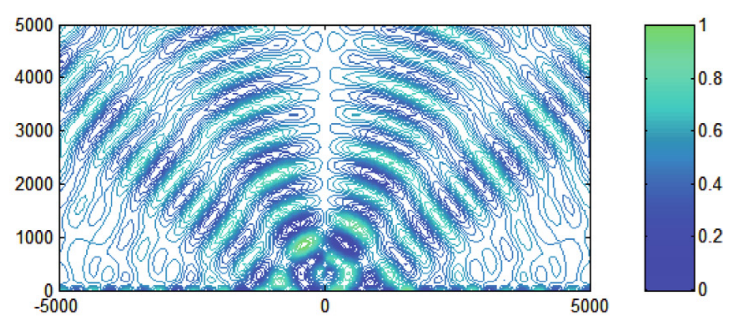

(d)

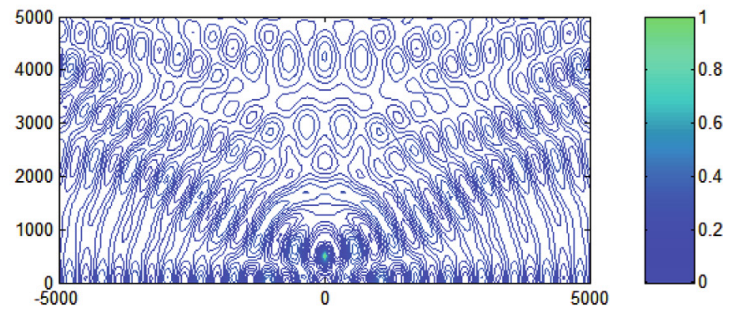

Figure 6. Components of $G$ (real part) with $Z=Z_{1}$ : (a) $G_{11}$, (b) $G_{12}$, (c) $G_{21}$, (d) $G_{22}$.

be analytically calculated. In effect, replacing $s=s^{*}$ in (4.1) and assuming $s_{L}<s^{*}<s_{T}$ gives

$$
\left(s_{T}^{2}-2 s^{* 2}\right)^{2}-\mathrm{i}\left(4 s^{* 2} \sqrt{s^{* 2}-s_{L}^{2}}-\frac{1}{\mu} s_{T}^{2} Z^{*}\right) \sqrt{s_{T}^{2}-s^{* 2}}=0 .
$$

Notice that the first term is real and the second term is pure imaginary. Hence, in order to fulfill equation (4.9), both terms have to vanish separately. The first term yields $s^{*}$, whereas the second term (together with the value of $s^{*}$ just obtained), gives $Z^{*}$. These values are

$$
s^{*}=\frac{\sqrt{2}}{2} s_{T}, \quad Z^{*}=2 \mu \sqrt{\frac{s_{T}^{2}}{2}-s_{L}^{2}} .
$$

Replacing the numerical values of $s_{T}, s_{L}$, and $\mu$ from (4.7) and (4.8) yields

$$
s^{*}=2.1112 \times 10^{-4}\left[\mathrm{~s} \cdot \mathrm{m}^{-1}\right], \quad Z^{*}=7.4421\left[\mathrm{MPa} \cdot \mathrm{s} \cdot \mathrm{m}^{-1}\right] .
$$

It is important to notice that when $Z$ approaches $Z^{*}$, the imaginary part of the additional pole becomes small (see Fig. 5b). This phenomenon produces abrupt variations in the spectral Green's function, which, although are not true singularities, they can cause numerical problems in computing the FFT. Therefore, even if the additional poles are complexes, it could be necessary to extract them in some cases.

\section{Numerical Results of the Green's function}

Next, we present some numerical results of the Green's function. The elastic material corresponds to limestone, and a frequency $f=5[\mathrm{~Hz}]$ is assumed, that is, a pulsation $\omega=2 \pi f=31.4159\left[\mathrm{rad} \cdot \mathrm{s}^{-1}\right]$. The source point $\boldsymbol{y}$ is fixed at $\left(y_{1}, y_{2}\right)=(0.500[\mathrm{~m}])$. The FFT is performed with $N=2^{16}$ points. Three cases were simulated, assuming $Z_{1}=0\left[\mathrm{MPa} \cdot \mathrm{s} \cdot \mathrm{m}^{-1}\right], Z_{2}=Z^{*}=7.4421\left[\mathrm{MPa} \cdot \mathrm{s} \cdot \mathrm{m}^{-1}\right]$, and $Z_{3}=60\left[\mathrm{MPa} \cdot \mathrm{s} \cdot \mathrm{m}^{-1}\right]$. The results are presented in Figures 6, 7, and 8, respectively. Each figure shows contour plots in $\boldsymbol{x}=\left(x_{1}, x_{2}\right)$ of each component of $G$ (real part). The numerical values have been scaled to $[0,1]$ in all cases. 
(a)

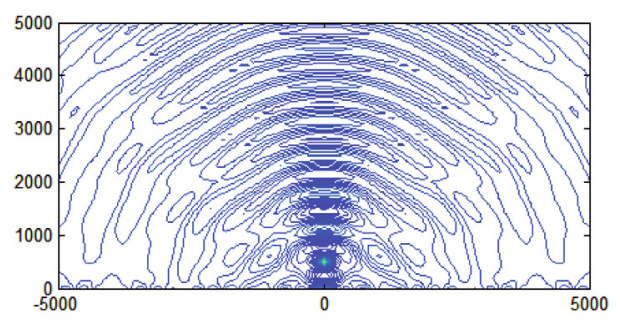

(c)

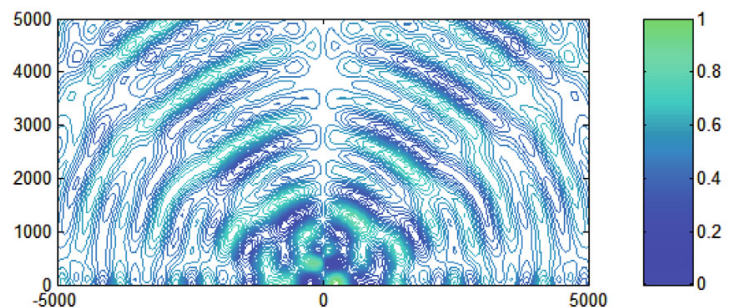

(b)

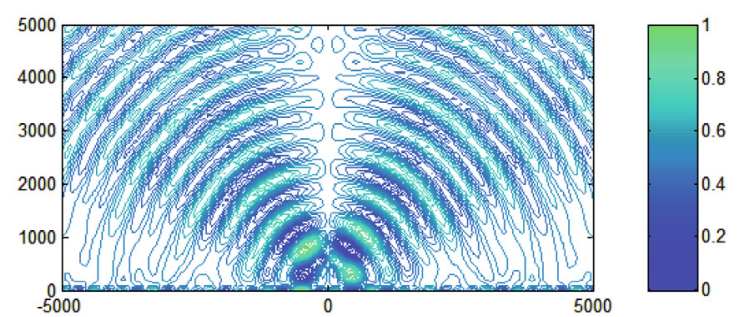

(d)

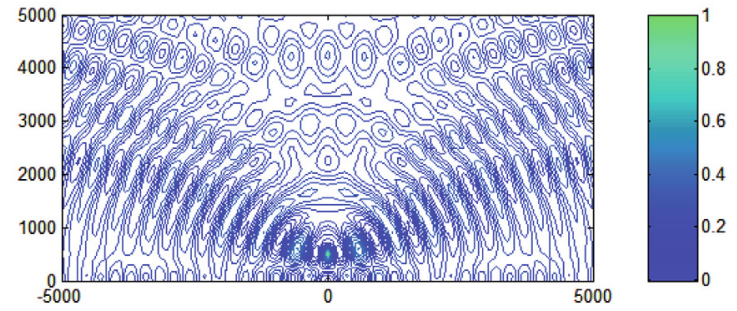

Figure 7. Components of $G$ (real part) with $Z=Z_{2}$ : (a) $G_{11}$, (b) $G_{12}$, (c) $G_{21}$, (d) $G_{22}$.

(a)

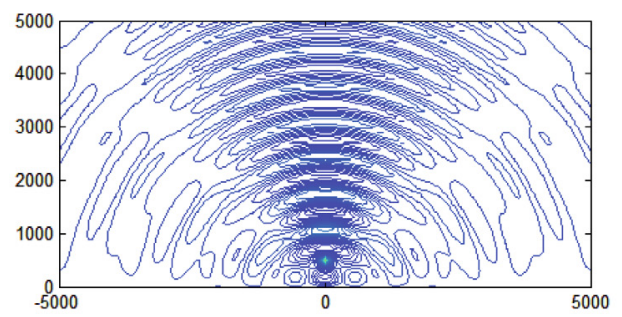

(c)

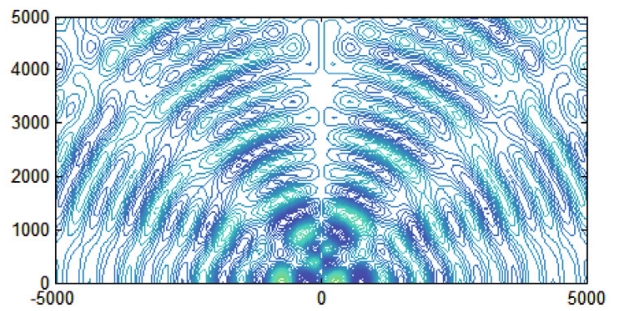

(b)

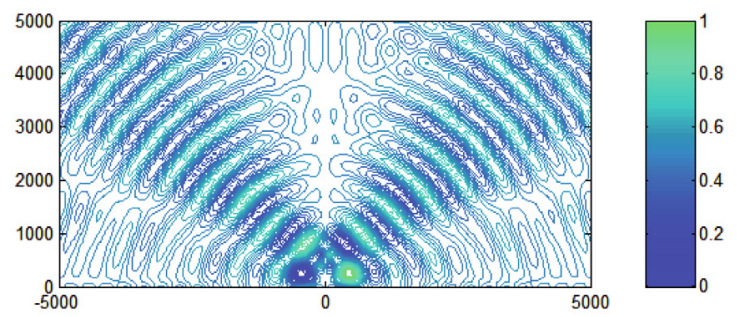

(d)
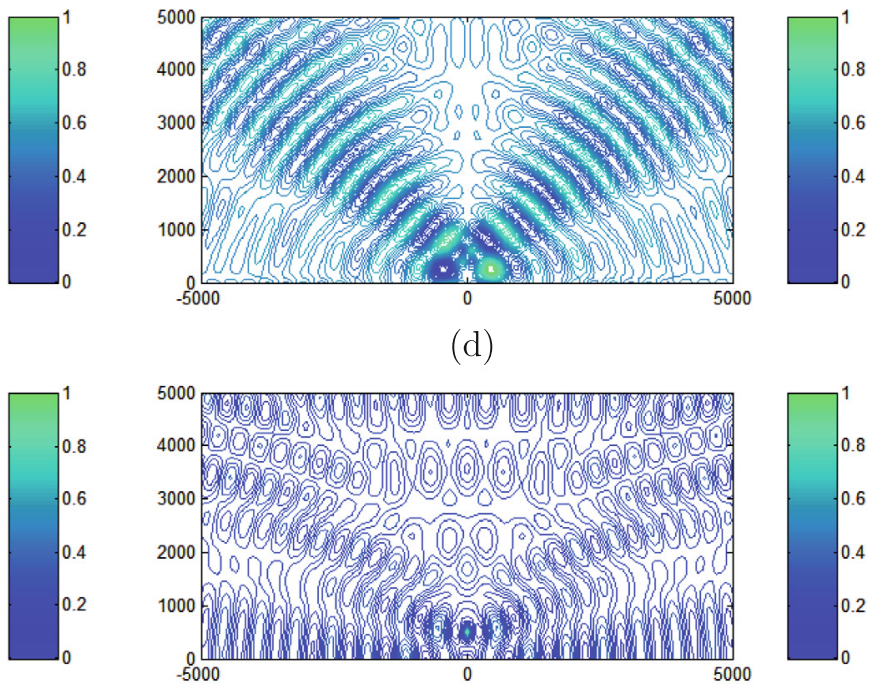

Figure 8. Components of $G$ (real part) with $Z=Z_{3}$ : (a) $G_{11}$, (b) $G_{12}$, (c) $G_{21}$, (d) $G_{22}$.

Acknowledgements. This work was partially supported by Proyecto ANILLO de Investigación ACT30 del Programa Bicentenario en Ciencia y Tecnología de CONICYT/World Bank (Chile) and the PUC fellowship for doctoral student. The authors want to thank Ignacio Muga for his support of this work. 


\section{REFERENCES}

[1] H. Bateman, Tables of Integral Transformations, Volume I. McGraw-Hill Book Company, Inc. (1954).

[2] W.W. Bell, Special Functions for Scientists and Engineers. Dover Publications, Inc., New York, USA (1968).

[3] M. Bonnet, Boundary Integral Equation Methods for Solids and Fluids. John Wiley \& Sons Ltd., Chichester, UK (1995).

[4] Z. Chen and M. Dravinski, Numerical evaluation of harmonic Green's functions for triclinic half-space with embedded sources Part I: A 2D model. Int. J. Numer. Meth. Engrg. 69 (2007) 347-366.

[5] Z. Chen and M. Dravinski, Numerical evaluation of harmonic Green's functions for triclinic half-space with embedded sources Part II: A 3D model. Int. J. Numer. Meth. Engrg. 69 (2007) 367-389.

[6] P. Colton and R. Kress, Integral Equations Methods in Scattering Theory. John Wiley, New York, USA (1983).

[7] J. Dompierre, Équations Intégrales en Axisymétrie Généralisée, Application à la Sismique Entre Puits. Ph.D. Thesis, École Centrale de Paris, France (1993).

[8] M. Durán, E. Godoy and J.-C. Nédélec, Computing Green's function of elasticity in a half-plane with impedance boundary condition. C. R. Acad. Sci. Paris, Ser. IIB 334 (2006) 725-731.

[9] M. Durán, I. Muga and J.-C. Nédélec, The Helmholtz equation in a locally perturbed half-plane with passive boundary. IMA J. Appl. Math. 71 (2006) 853-876.

[10] M. Durán, R. Hein and J.-C. Nédélec, Computing numerically the Green's function of the half-plane Helmholtz operator with impedance boundary conditions. Numer. Math. 107 (2007) 295-314.

[11] G.R. Franssens, Calculation of the elasto-dynamics Green's function in layered media by means of a modified propagator matrix method. Geophys. J. Roy. Astro. Soc. 75 (1983) 669-691.

[12] F.B. Jensen, W.A. Kuperman, M.B. Porter and H. Schmidt, Computational Ocean Acoustics. Springer-Verlag, New York, USA (1994).

[13] L.R. Johnson, Green function's for Lamb's Problem. Geophys. J. Roy. Astro. Soc. 37 (1974) 99-131.

[14] A.M. Linkov, Boundary Integral Equations in Elasticity Theory. Kluwer Academic Publishers, Dordrecht, Boston (2002).

[15] A.M. Linkov, A theory of rupture pulse on softening interface with application to the Chi-Chi earthquake. J. Geophys. Res. 111 (2006) 1-14.

[16] J.-C. Nédélec, Acoustic and Electromagnetic Equations - Integral Representations for Harmonic Problems, Applied Mathematical Sciences 144. Springer, Germany (2001).

[17] C. Richter and G. Schmid, A Green's function time-domain boundary element method for the elastodynamic half-plane. Int. J. Numer. Meth. Engrg. 46 (1999) 627-648.

[18] M. Spies, Green's tensor function for Lamb's problem: The general anisotropic case. J. Acoust. Soc. Am. 102 (1997) $2438-2441$.

[19] T.R. Stacey and C.H. Page, Practical Handbook for Underground Rock Mechanics, Series on Rock and Soil Mechanics 12. Trans Tech Publications, Germany (1986).

[20] C.-Y. Wang and J.D. Achenbach, Lamb's problem for solids of general anisotropy. Wave Mot. 24 (1996) $227-242$. 